\title{
High Brightness Photo Injectors for Brilliant Light Sources
}

\author{
Frank Stephan* and Mikhail Krasilnikov \\ Deutsches Elektronen Synchrotron DESY, Zeuthen, Germany
}

Keywords Average current - Beam optimization $\bullet$ Beam quality $\bullet$ Brightness $\bullet$ DC guns $\bullet$ Emittance $\bullet$ Photo injectors $\bullet$ RF guns

\begin{abstract}
The large variety of existing and planned light sources sets specific demands on the performance of the corresponding electron injectors which requires specific solutions. In the introductory part of this chapter, a general injector layout and three types of photo emission-based electron sources will be described. In subsequent sections, different designs of electron sources providing a wide range of average electron currents from $\mathrm{nA}$ to $100 \mathrm{~mA}$ will be discussed. Basics of the experimental optimization of modern electron sources and future options will be presented.
\end{abstract}

\section{Introduction}

The development and reliable operation of high brightness electron sources are key issues for current and next-generation short wavelength light sources. But there is no universal solution to build electron sources for all high brightness beam applications. Different applications like short wavelength self-amplified spontaneous emission-free electron lasers (SASE FELs) or energy recovery linacs (ERLs) set different stringent requirements on the electron source which forces different technical realizations. Some key parameters for the source development, which in most cases are defined by the users of the corresponding facility and the technology choice for the subsequent accelerator (e.g., normal conducting or superconducting), are:

- Mode of operation: pulsed or (quasi-)continuous wave (CW) operation

- Single bunch charge

- Time structure of the beam: single pulses or pulse trains, single bunch repetition rate, length of the pulse trains, and pulse train repetition rate

- Normalized transverse emittance required for the application

- Whether or not further bunch compression is needed after the source, required longitudinal phase-space parameters at the application

- Stability requirements, shot-to-short and within possible pulse trains

Beyond setting the definition for the injector setup, these parameters require a sophisticated design from the electron source to the application of the beam, e.g., the undulator of an FEL.

*E-mail: frank.stephan@desy.de 
To maximize the single bunch brightness $\mathscr{B}$ (Piot 2006), one would like to concentrate a maximum of charge $Q$ in the smallest possible $6 \mathrm{D}$ phase-space volume ${ }^{1}$

$$
\mathscr{B}=\frac{Q}{\epsilon_{n, x} \epsilon_{n, y} \epsilon_{n, z}}
$$

where $\epsilon_{n, u}, u \in\{x, y, z\}$ is the normalized RMS emittance in all three coordinate planes. In order to generate high charge without strong deterioration of the transverse phase space $\left(\epsilon_{n, x}, \epsilon_{n, y}\right)$ due to space charge effects (Coulomb repulsion), longer electron bunches from the source (possibly including corresponding temporal laser pulse shaping) and fast acceleration of the bunch are required. Due to the fast acceleration, the electron bunch length settles on a constant value soon after the photo emission when propagating in free space. This would allow that the ratio $Q / \epsilon_{n, z}$ could be approximated with the bunch current which is the ratio of bunch charge and bunch length. However, the parameters of the longitudinal phase space might be changed significantly by accelerator elements further downstream of the electron source. For example, the bunch length, peak current, energy spread, and longitudinal emittance will change when the bunch is compressed or higher harmonic RF accelerating systems for linearizing the longitudinal phase space are applied at a higher energy level, as will be shown later. Since the calculation of a single bunch peak brightness requires the usage of peak current, which is not constant along the accelerator, the peak brightness of the electron beam will not be used as a figure of merit in this chapter. Instead, the beam quality will be characterized by the measured or designed normalized transverse emittance at a certain beam energy level for a given bunch charge.

To characterize the single bunch brightness, the weighted average slice brightness $\left\langle B_{\text {slice }}\right\rangle_{w}$ can be defined where the slice brightness $B_{\text {slice }}(t)$ is weighted with the corresponding slice current:

$$
\begin{array}{rr}
B_{\text {slice }}(t)=\frac{I_{\text {slice }}(t)}{\epsilon_{n, x, \text { slice }}(t) \cdot \epsilon_{n, y, \text { slice }}(t)} & {\left[\mathrm{A} /(\mathrm{mm} \mathrm{mrad})^{2}\right]} \\
\left\langle B_{\text {slice }}\right\rangle_{w}=\int I_{\text {slice }}(t) \cdot B_{\text {slice }}(t) d t / \int I_{\text {slice }}(t) d t & {\left[\mathrm{~A} /(\mathrm{mm} \mathrm{mrad})^{2}\right]}
\end{array}
$$

Here $I_{\text {slice }}(t)$ is the slice current defined by the charge in the temporal slice divided by its length and $\epsilon_{n, x \text {,slice }}(t)$ and $\epsilon_{n, y, \text { slice }}(t)$ are the horizontal and vertical normalized slice emittances, respectively. For the mathematical definition of slice current, slice emittance, and slice brightness, the time interval of the slice length is infinitesimally small so that $I(t), \epsilon_{n, x}(t), \epsilon_{n, y}(t)$, and $B(t)$ are continuous quantities. This definition will be used in section "Generic Photo Injector Layout and Beam Dynamics Simulations" where different photo cathode laser shapes and correspondingly different electron bunch shapes are studied. On the other hand, practical measurements of the slice properties are always integrated over a certain slice length, that is, the resolution of the slice diagnostics. Although this seems to be an unfortunate fact at first glance, it can be of advantage for defining useful quantities which characterize the usable beam quality if the time interval of the slice, the time resolution, is not too coarse. For example, for the SASE FEL process, the relevant time interval is the coherence time (Schmüser, Dohlus and Rossbach 2008). For slice

\footnotetext{
${ }^{1}$ Equation 1 assumes that there is no significant coupling between the transverse phase spaces and between the transverse and the longitudinal phase spaces.
} 
diagnostics capable of resolving this time interval, the beam quality relevant for the SASE FEL process can be measured, while a measurement and definition of the beam brightness at much better time resolution does not characterize the amplification process anymore since this would neglect a possible mismatch of the different slices during their interaction in the SASE FEL process. Therefore, for practical use, the slice parameters always have to be defined on the time scale which is relevant for the underlying physics process that the beam is applied for.

Additionally, several applications require not only good bunch properties but also a high average beam current. For those the average brightness of the injector $B_{\text {injector }}$ being defined as the total charge generated per second divided by the normalized projected transverse emittances is a useful quantity $^{2}$ :

$$
B_{\text {injector }}=Q_{\text {bunch }} \cdot \mathrm{NoP} \cdot \mathrm{RR} /\left(\epsilon_{n, x} \cdot \epsilon_{n, y}\right) \quad\left[\mathrm{A} /(\mathrm{mm} \mathrm{mrad})^{2}\right]
$$

Here $Q_{\text {bunch }}$ is the single bunch charge, NoP is the number of pulses generated within a possible bunch train, $\mathrm{RR}$ is the repetition rate (of the pulse train), and $\epsilon_{n, x}$ and $\epsilon_{n, y}$ are the horizontal and vertical normalized projected emittances of the individual bunches, respectively. This average brightness of the injector will be used as an additional figure of merit to compare different electron sources.

In the next subsection, a generic injector layout as it is used for many photo injector applications will be described. This will include simulations for different photo cathode laser shapes as well as a description of the optimization procedure used for the simulations. In the subsequent subsections, the three basic types of photoelectron sources will be briefly defined, and their basic advantages and disadvantages will be discussed.

After this introductory part, different photo injector projects are presented, their parameter sets are listed, and their challenges are described briefly. The parameter range for bunch charges is between $10 \mathrm{fC}$ and $7 \mathrm{nC}$, the time structure ranges from $10 \mathrm{~Hz}$ single bunches to $1.3 \mathrm{GHz} \mathrm{CW}$ operation, and the measured or designed normalized emittances range from 0.1 to $\sim 10 \mathrm{~mm} \mathrm{mrad}$. The average currents from the injector $I_{\text {injector }}=Q_{\text {bunch }} \cdot \mathrm{NoP} \cdot \mathrm{RR}$ for the different projects range over approximately eight orders of magnitude, from nano-Amperes up to currents of $100 \mathrm{~mA}$. The different projects are grouped into three categories: high, medium, and low average current for $I_{\text {injector }} \geq 1 \mathrm{~mA}, 1 \mathrm{~mA}>I_{\text {injector }}>1 \mu \mathrm{A}$, and $I_{\text {injector }} \leq 1 \mu \mathrm{A}$, respectively.

The selected photo injector projects are by no means exhaustive and simply represent a personal selection of interesting projects on the basis of which specific problems and solutions relating to photo injector developments can be discussed.

After the description and comparison of different photo injector projects, section "Comparing Photo Injector Performances and Future Trends" will summarize some future directions for the further development of photo injectors, e.g., special shaping of the photocathode laser pulses to obtain 3D elliptical electron bunch distributions, the development of photo cathodes operating for green laser light, and also a new idea to generate high brightness electron beams inside the high accelerating field of a plasma acceleration bubble.

Throughout this chapter, emittance is always discussed as normalized transverse emittance if not explicitly stated otherwise.

\footnotetext{
${ }^{2}$ For Eq. 4 constant charges and projected transverse emittances of the bunches are assumed over all bunches generated within $1 \mathrm{~s}$. This is not necessarily straightforward since it needs feedback algorithms to stabilize the RF and photo cathode laser properties. In addition it should be noted that the average injector brightness is not the sum of the average slice brightness of all bunches per second, because the average slice brightness of each bunch does not include the mismatch between the different slices of the bunch.
} 


\section{Generic Photo Injector Layout and Beam Dynamics Simulations}

\section{Basic Principles}

In order to construct a high brightness electron beam injector, several general design considerations have to be applied; see, e.g., Ferrario et al. (2000, 2001).

Typically a photo injector consists of a photo gun and a booster cavity. At the beginning, the longitudinal bunch profile and the temporal bunch pattern of the accelerator are generated by illuminating the photocathode with appropriate photocathode laser pulses. Electromagnetic fields (or electric fields in the case of DC guns) are applied to accelerate the bunch, which has to happen as quickly as possible to mitigate space charge effects. This is why the accelerating electric field at the emission point has to be as high as possible. On the other hand, the photocathode surface roughness will play a greater role at high cathode gradients and can dilute the intrinsic emittance (Krasilnikov 2006; Xiang et al. 2007), often also called thermal emittance. This can result in an optimum peak field at the cathode delivering the minimum overall emittance. In earlier times, it was expected that higher-frequency RF guns would be the best choice for obtaining minimum emittance since they can provide higher cathode gradients. But they have their own systematic limitations like, for example, RF-induced emittance growth for long photocathode laser pulses and high heat load on the surface causing restrictions on RF pulse length and repetition rate as well as long-term reliability issues. Now, the examples listed in this chapter show that over a wide range of accelerating gradients at the cathode, good beam properties can be demonstrated when the overall optimization of the full photo injector is done carefully. Nevertheless, increasing the cathode gradient is highly desired and remains a technological challenge.

To focus the electron beam transversely, a static magnetic focusing system, typically based on solenoids, is used. The solenoid field is also used to counteract the space charge-induced emittance growth. This process is called emittance compensation (Carlsten 1989, 1995; Serafini and Rosenzweig 1997). That is why the solenoid field in most cases is applied either around the gun cavity itself or shortly downstream. The emittance compensation process is mainly provided by a good balance of the space charge forces and external focusing from properly tuned solenoid and RF fields. The space charge force introduces nonlinear correlations in the transverse phase space along the electron bunch. The solenoid field reverses the space charge action, resulting in a better alignment of the temporally sliced phase spaces and therefore in a minimum projected emittance. Further drift of the electron beam will result in emittance growth due to the space charge influence onto the transverse phase space, increasing the difference in the phase-space correlations along the bunch. In order to prevent this growth, further acceleration has to be applied.

The energy gain in the gun is usually not sufficient to maintain the good beam quality over long distances and to prevent space charge-induced emittance growth further downstream. To exit the space-charge dominated regime of the low-energy electron beam (few $\mathrm{MeV}$ ), a subsequent accelerating section - a booster - is applied to increase the beam energy to a few tens or even a few hundreds of MeV. For this so-called emittance conservation process, special care has to be taken to place the booster in the appropriate position and apply the appropriate accelerating gradient and RF phase to the low-energy beam following the laminarity condition (Serafini and Rosenzweig 1997; Ferrario et al. 2000). For this so-called invariant envelope technique, the laminar waist of the beam should be around the booster entrance, and the accelerating gradient should provide simultaneous reduction of the beam spot size and the transverse momentum. This final stage of the emittance compensation process will conserve and even further improve the beam quality originating from the source. Sometimes a part of the booster or additional cavities are also used to introduce an 
energy chirp to realize ballistic bunching (Serafini and Ferrario 2001) which increases the bunch current already in the injector.

Besides a careful design on the usage of different accelerating cavities and the beam focusing system, also the individual laser pulses illuminating the photocathode have to be appropriately shaped in transverse space as well as temporally in order to generate minimum emittance beams. Since the photocathode laser pulse determines the initial properties of the electron bunch in a photo injector and the first emittance growth already takes place during the electron emission and the first millimeters of beam propagation, the full 6D beam dynamics has to be considered at the photocathode. The final goal is to produce a high charge, i.e., a high number of electrons, enclosed in a smallest possible volume in phase space. An increase of the transverse laser spot size helps to produce higher bunch charges, but this also leads to an increase of the thermal emittance, a minimum threshold emittance already defined at the photo cathode, which is proportional to the photocathode laser spot size. In addition, when assuming that the phase-space density is kept constant, an increase of the laser spot size also results in a higher contribution from the transverse space charge nonlinearities in the cathode vicinity, e.g., when generating pancake-like beam shapes during the emission. On the other hand, an increase of the temporal duration of the photocathode laser pulse allows higher charge production and relaxes the space charge impact onto the transverse phase space, but at the same time the longitudinal emittance increases. Therefore, the optimization of the photocathode laser pulses is a reasonable compromise between longitudinal and transverse laser beam shaping.

Simulation studies have shown (see, e.g., Ferrario et al. 2001) that fairly long laser pulses should be applied at the photocathode if very low normalized emittances are required. The long electron pulses acquire some RF curvature during the acceleration in the gun and in the booster which increases the longitudinal emittance and can produce a spiked current distribution with strong coherent synchrotron radiation (CSR) effects during compression of the beam in a bunch compressor. In order to prevent this, a higher harmonic cavity can be applied to linearize the longitudinal phase space upstream of the bunch compressor section which then results in a more efficient compression (Flöttmann et al. 2001). But wakefields which are strongly dependent on the individual geometry of the vacuum components have to be kept under control as well to perform a good bunch compression.

Since the uncorrelated (slice) energy spread of electron bunches generated from the photo injector is very small, the microbunching instability (Saldin et al. 2002; Huang et al. 2004) may lead to a significant amplification of the longitudinal phase space modulations originating from the RF gun or even from the shot noise of the electron beam. The uncorrelated energy spread can be increased significantly to provide a strong Landau damping against the instability without degrading the high brightness photo injector performance (Huang et al. 2004, 2009). Currently the laser heater is considered as one of the most efficient tools to minimize the impact of the microbunching instability on the quality of the electron beam which has been proven experimentally (Huang et al. 2009).

All abovementioned effects contribute to the overall transverse emittance budget:

$$
\varepsilon_{\mathrm{tot}}=\sqrt{\varepsilon_{\mathrm{th}}^{2}+\varepsilon_{\mathrm{RF}}^{2}+\varepsilon_{\mathrm{SC}}^{2}}
$$

where the initial thermal emittance $\varepsilon_{\text {th }}$, the RF-induced $\varepsilon_{\mathrm{RF}}$, and the space charge-induced $\varepsilon_{\mathrm{SC}}$ emittances are added in quadrature. Here a correlation term that describes the possible interaction between the space charge field and RF forces is neglected. A simple modeling of the emission 
results in the dependence $\varepsilon_{\mathrm{th}, x, y} \sim \sigma_{x, y} \sqrt{E_{k}}$ (Floettmann 1997; Dowell and Smerge 2009), where $\sigma_{x, y}$ is the initial RMS transverse size of the beam at the cathode and $E_{k}$ is related to the mean kinetic energy of the emitted particles. This contribution is uncorrelated and often serves as a lower limit of the achievable emittance minimum. The emittance contribution due to the RF effect scales as $\varepsilon_{\mathrm{RF}} \sim \sigma_{x, y}^{2} \sigma_{z}^{2}$ (Kim 1989), where the electron bunch length $\sigma_{z}$ is involved. The space chargeinduced emittance $\varepsilon_{\mathrm{SC}}$ is the most complex contribution for the modeling and is mainly a subject for numerical optimization.

Within the photo injector setup, different diagnostics devices are essential to measure the drive laser and electron beam properties in detail. Downstream of the injector, the electron beam will in many cases obtain further acceleration and will pass through bunch compression stages before it arrives at its application, e.g., a SASE FEL.

\section{Beam Dynamics Simulations for the European XFEL Photo Injector}

A typical layout of a high brightness photo injector is shown in Fig. 1 where the principal scheme of the photo injector of the European X-ray Free Electron Laser (European XFEL) is presented. It consists of a photocathode RF gun supplied with solenoid magnets for emittance compensation, one accelerator module with eight superconducting 9-cell TESLA-type cavities, and a 3rd harmonic linearizer followed by a diagnostic section with a laser heater. Downstream of a dogleg, the first bunch compressor is located. The first half of the accelerating module (first four cavities in the TESLA module in Fig. 1) serves as a booster with matched accelerating gradients and phases to follow the emittance conservation principle. The cavities in the second half of the TESLA module run at high accelerating gradient to obtain a high-energy gain.

To find the working point of the high brightness photo injector is a multiparametric complex task, where the many injector parameters, e.g., cathode laser pulse transverse and longitudinal profile; RF gradient and emission phase in the gun; solenoid strength and location; booster position, gradient, and phase; higher harmonic acceleration section; and bunch compressor parameters, with their many interdependences have to be optimized.

As an example for such a multivariate injector optimization, the beam dynamics in the photo injector of the European XFEL was simulated using the ASTRA code (ASTRA Manual 2014). Cylindrical photocathode laser pulses with a flattop temporal profile of 21.5 ps FWHM and 2 ps rise and fall time and a radially homogeneous transverse distribution with $0.41 \mathrm{~mm}$ RMS spot size are considered as the nominal case for the European XFEL photo injector for the operation with $1 \mathrm{nC}$ bunch charge. An accelerating electric RF field with an amplitude of $60.6 \mathrm{MV} / \mathrm{m}$ at the cathode and an initial kinetic energy of the photo-emitted electrons of $0.55 \mathrm{eV}$ are assumed at the $\mathrm{Cs}_{2} \mathrm{Te}$ photo cathode. These gun parameters correspond to the optimum experimental performance demonstrated at the photo injector test facility at DESY, Zeuthen site (PITZ) (Krasilnikov et al. 2012).

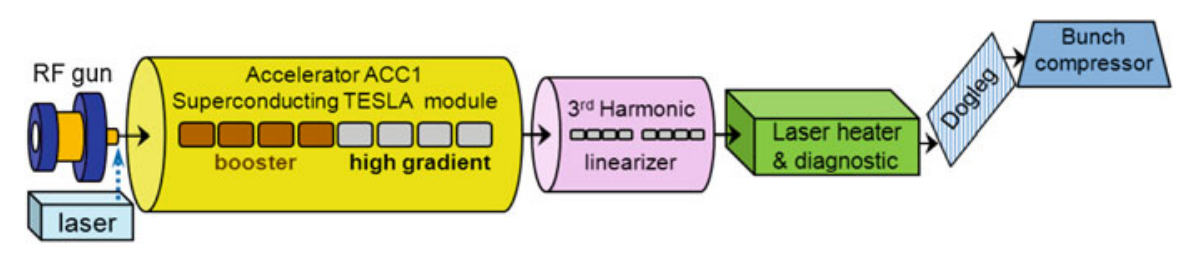

Fig. 1 Schematic layout of the European XFEL injector 

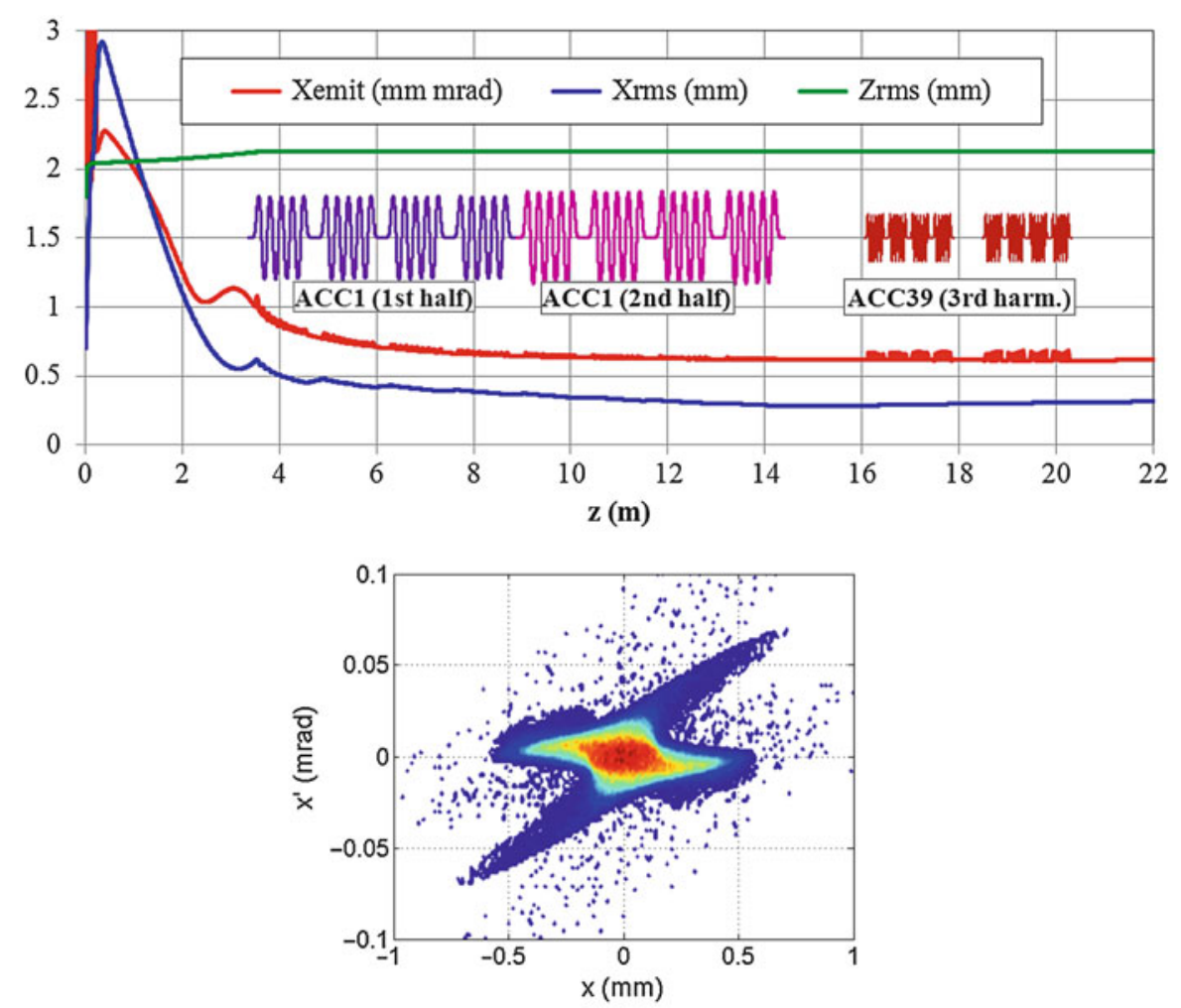

Fig. 2 Upper plot: normalized projected transverse emittance (Xemit), RMS horizontal beam size (Xrms), and RMS bunch length (Zrms) as a function of the distance from the photocathode at the European XFEL injector. Lower plot: transverse phase space of the electron beam at $z=15 \mathrm{~m}$

The first half of the first accelerating module (ACC1) serves as a booster with a maximum RF electric field on-crest of $30.2 \mathrm{MV} / \mathrm{m}$. The second half is also operated on-crest but with a maximum field of $33.5 \mathrm{MV} / \mathrm{m}$, delivering a total beam energy of $\sim 143 \mathrm{MeV}$ at the ACC1 exit. The main goal of the optimization was a minimum transverse normalized projected emittance at $z=15 \mathrm{~m}$, i.e., downstream of ACC1. The resulting evolution of the electron beam parameters along the photo injector is shown in Figs. 2 and 3 (upper plots).

Figure 2 (upper plot) illustrates the typical projected emittance oscillation in the gun as well as the proper matching of the booster with the incoming electron beam. The simulated transverse phase space of the electron beam after the first accelerating module is shown at the lower plot of the Fig. 2.

The applied cathode laser pulse duration ( 21.5 ps FWHM) corresponds to about $11 \mathrm{deg}$ of the RF period, and a longitudinal emittance growth is introduced by the cosine-like RF field due to the fact that the electron bunch length occupies a significant fraction of the RF wavelength. Such a nonlinearity of the longitudinal phase space complicates the bunch compression in subsequent magnetic chicanes. In order to remove these nonlinear correlations, a third-order accelerating cavity (3rd harmonic linearizer in Fig. 1) is applied. In Fig. 3, the strong reduction of the longitudinal emittance by using the third harmonic cavity is visible. The third-harmonic section also provides possibilities for pre-compensating nonlinearities of the subsequent bunch compressor as well as collective effects like longitudinal wake fields and coherent synchrotron radiation. The effect of the nonlinear correction is illustrated in the lower plot of Fig. 3 where the longitudinal phase space before and after the third harmonics section is shown. 

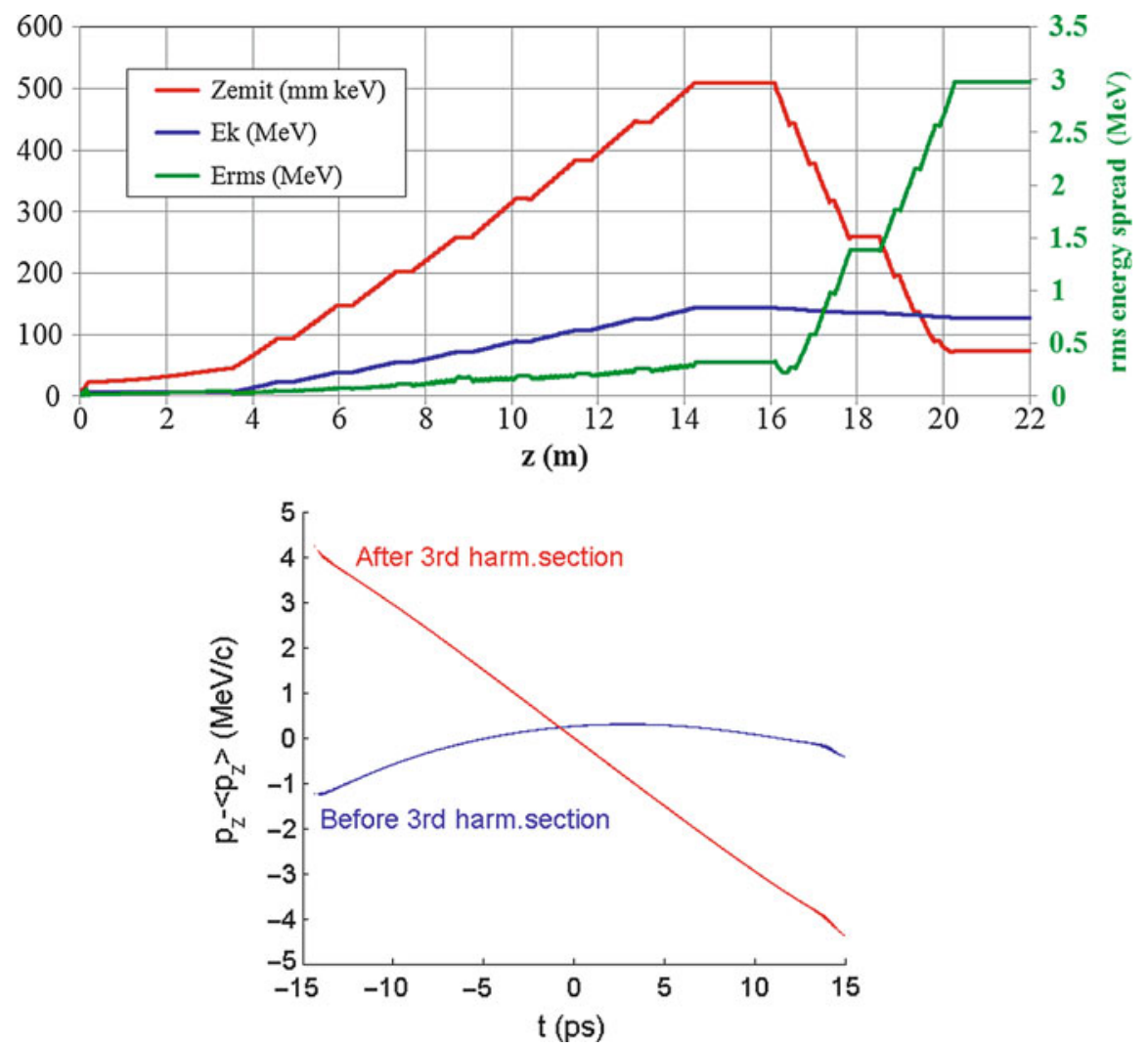

Fig. 3 Upper plot: longitudinal emittance (Zemit), mean beam energy (Ek), and RMS energy spread (Erms, right axis) as a function of the distance from the photocathode for flattop photocathode laser pulses at the European XFEL injector. Lower plot: longitudinal phase space before (blue) and after (red) the third harmonics linearizer

\section{Various Shapes of the Photocathode Laser Pulse}

A proper cathode laser pulse shaping is one of the major instruments which helps to reduce the space charge-induced contribution to the overall emittance budget. The space charge effect during photo emission of a high-density electron bunch plays an important role in the slice emittance formation introducing transverse phase-space nonlinearities and nonlinear correlations along the bunch. Application of a flattop temporal profile yields a significant improvement of the beam emittance in comparison to a Gaussian temporal distribution because the charge density in the center of the bunch is lower and more homogeneously distributed along the bunch, resulting in a more linear space charge force distribution. Further improvement can be achieved by applying 3D ellipsoidal laser pulses. Whereas Gaussian and flattop temporal profile laser pulses have a transverse cylindrical shape, a 3D ellipsoidal pulse distribution corresponds to a homogeneously filled ellipsoid, i.e., the local border radius depends on the temporal position within the laser pulse. In this case, the produced electron beam is closer to a charge configuration corresponding to linear space charge distributions in all directions.

The beam dynamics optimizations for all cases were performed to deliver the same RMS length of the electron bunch and minimizing the transverse normalized projected emittance at $z=15 \mathrm{~m}$, the exit of ACC1. The optimized photo injector parameters are summarized in Table 1 for three shapes of the photocathode laser pulses together with the obtained electron bunch properties after the first accelerating module. The other parameters - photo injector layout, RF gradient at the 
Table 1 Optimized simulated photo injector parameters for various shapes of the photocathode laser pulse

\begin{tabular}{|c|c|c|c|c|}
\hline Parameter & Unit & Gaussian & Flattop & 3D Ellipsoid \\
\hline \multicolumn{5}{|c|}{ Optimized photo injector parameters } \\
\hline Laser $\sigma_{x y}^{l \text { a }}$ & $\mathrm{mm}$ & 0.39 & 0.42 & 0.40 \\
\hline Laser $\sigma_{t}^{l \mathrm{~b}}$ & ps & 5.29 & 6.27 & 6.00 \\
\hline Gun phase $^{\mathrm{c}}$ & deg & -2.33 & -1.50 & -2.29 \\
\hline$B_{z, \text { solenoid }}^{\text {peak }}{ }^{\mathrm{d}}$ & $\mathrm{mT}$ & 226.9 & 227.5 & 229.5 \\
\hline
\end{tabular}

Electron beam at $\mathrm{z}=15 \mathrm{~m}$

\begin{tabular}{l|l|c|c|c}
\hline$\varepsilon_{x}^{\text {projected }}$ & $\mathrm{mm} \mathrm{mrad}$ & 1.05 & 0.62 & 0.43 \\
\hline Peak current & $\mathrm{A}$ & 46.0 & 43.8 & 47.3 \\
\hline$\left\langle\varepsilon_{x}^{\text {slice }}\right\rangle^{\mathrm{e}}$ & $\mathrm{mm} \mathrm{mrad}$ & 0.72 & 0.55 & 0.40 \\
\hline$\left\langle B_{\text {slice }}\right\rangle^{\mathrm{e}}$ & $\frac{A}{(\mathrm{~mm} \mathrm{mrad})^{2}}$ & 90 & 135 & 240 \\
\hline
\end{tabular}

${ }^{\mathrm{a}} \mathrm{RMS}$ size of the round laser spot $\sigma_{x y}^{l}=\sqrt{\sigma_{x}^{l} \sigma_{y}^{l}}=\sigma_{x}^{l}=\sigma_{y}^{l}$

${ }^{\mathrm{b}}$ The RMS pulse duration was tuned to generate electron bunches with RMS length of $\sigma_{z}=2.13 \mathrm{~mm}$ at $z=15 \mathrm{~m}$. The flattop pulse with 21.5 ps FWHM and 2 ps rise/fall time is used as a reference

${ }^{\mathrm{c}}$ W.r.t. the phase of the maximum mean momentum gain

${ }^{\mathrm{d}}$ Peak field of the main solenoid. The bucking solenoid was always tuned to compensate the longitudinal magnetic field at the cathode

${ }^{\mathrm{e}}$ The averaged weighted values within the bunch

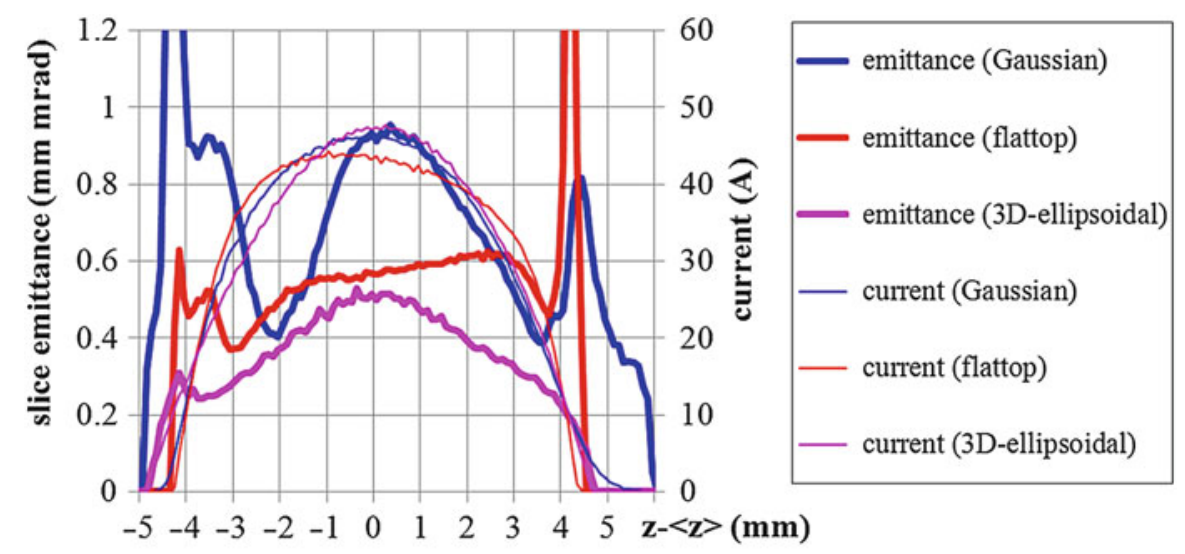

Fig. 4 Simulated slice emittance and slice current at the exit of the first acceleration module of the European XFEL $(z=15 \mathrm{~m})$ for 3 different photocathode laser pulse shapes: Gaussian, flattop, 3D ellipsoidal

cathode, initial kinetic energy of the emitted electrons, ACC1 gradients and phases - were fixed to the optimum values found for the flattop case as discussed in section "Beam Dynamics Simulations for the European XFEL Photo Injector."

The results of corresponding beam dynamics simulations for the European XFEL photo injector are shown in Fig. 4, where the slice emittance of electron bunches produced by various shapes of the photocathode laser pulses are presented together with the corresponding beam current profiles. One clearly sees a higher space charge density in the center and a lower density in the head and tail of the bunch for the Gaussian case resulting in an increased slice emittance in the center as well as in the head and tail of the bunch. A flattop laser pulse generates electron bunches with a more homogeneous slice emittance profile, but still with spikes in the head and tail. This is due to nonlinearities in the transition regions between flattop and flanks. A much smoother slice emittance distribution is obtained by applying a 3D ellipsoidal cathode laser pulse. 


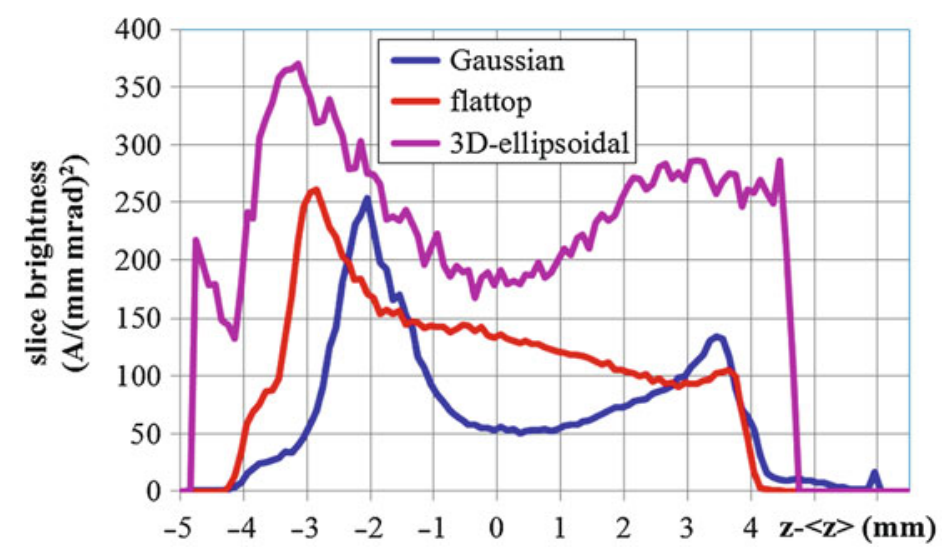

Fig. 5 Simulated slice brightness at the exit of the first acceleration module of the European XFEL $(z=15 \mathrm{~m})$ for 3 different photocathode laser pulse shapes: Gaussian, flattop, 3D ellipsoidal

The overall shape of the slice emittance curve contains a large fraction of the noncorrelated thermal (intrinsic cathode) emittance which is mainly determined by the local beam diameter. The average (weighted) slice emittance for the case of flattop pulses is $0.55 \mathrm{~mm}$ mrad which is $\sim 24 \%$ smaller than obtained for the Gaussian pulses. 3D ellipsoidal cathode laser pulses generate electron bunches with an average slice emittance of $0.40 \mathrm{~mm}$ mrad which is a further $\sim 27 \%$ reduction w.r.t. the flattop case.

A similar trend is obtained from the comparison of the slice brightness (Eq. 2). Corresponding slice brightness distributions for the three abovementioned cathode laser pulse shapes are shown in Fig. 5. Comparing the average slice brightness (Eq.3) demonstrates a $50 \%$ improvement by applying flattop pulses w.r.t. the Gaussian ones. The usage of 3D ellipsoidal cathode laser pulses results in an increase of the average slice brightness by almost another $80 \%$ compared to the flattop case.

\section{Different Types of Photo Injectors}

\section{Photoelectron Guns}

In DC photoelectron guns, the photocathode is placed at a static negative potential of several $100 \mathrm{kV}$. Because of the specific geometry of cathode and anode and their separation, the accelerating field at the cathode is of the order of a few $\mathrm{MV} / \mathrm{m}$. Three important advantages of DC photoelectron guns are (a) the good vacuum conditions with pressures down to $10^{-12}$ mbar, (b) the rich operating experience in many different applications, and (c) the large flexibility regarding the temporal bunch pattern which is only restricted by the photocathode laser capabilities, beam dynamics limitations, and the subsequent RF acceleration. Because of the good vacuum environment, negative electron affinity (NEA) cathodes like, e.g., GaAs can be used in DC photoelectron guns. If illuminated with the proper laser wavelength, this material has been shown to result in a low kinetic energy of the photo-emitted electrons at the cathode providing a low thermal emittance for a given laser spot size. For early measurements of GaAs cathodes, a transverse momentum corresponding to a thermal energy of $34 \mathrm{meV}$ was reported (Dunham et al. 1995). More recent measurements at $90 \mathrm{~K}$ showed a most probable transverse momentum corresponding to a kinetic energy of the emitted electrons of about $10 \mathrm{meV}$ (Orlov et al. 2004). 
Two clear disadvantages of DC photoelectron guns are the relatively low accelerating gradient at the cathode and the low energy of the electrons at the exit of the DC gun. In order to reduce the space charge force and overcome the space charge limit for charge extraction from the cathode, the emitting laser pulse is either large in diameter or long in time. Since an increased transverse laser spot size results in an increased thermal emittance, in general, the laser pulse length is increased for extracting significant bunch charges. To accommodate later RF acceleration, the long electron bunches are usually compressed at low energy with low-frequency buncher cavities, which can result in a deterioration of the transverse emittance. Newer developments in this respect will be mentioned in section "DC Photocathode Gun Development at Cornell." After the buncher cavity, the electron beam still has low momentum and therefore requires further booster acceleration with proper matching of the beam.

\section{Normal Conducting RF Guns}

In this kind of electron source, the photocathode is located at the backplane of a normal conducting (NC) RF cavity. The first cell usually has a reduced length (half cell) so that a high accelerating surface field is obtained at the cathode. The cavity is supplied with a solenoid magnet for space charge compensation; see section "Generic Photo Injector Layout and Beam Dynamics Simulations." In some cases, a bucking solenoid in the rear of the cathode plane is needed to zero the magnetic field of the main solenoid on the cathode. The high accelerating gradient at the cathode, the subsequent accelerating cells, and the solenoid arrangement for the space charge compensation allow operation of this electron source with a high bunch charge as well as higher beam energy from the source as compared to DC guns. A wealth of experience has been gathered for operating NC RF guns at many different locations, and this type of electron source has experimentally proven to provide the best emittance results for given bunch charge; see section "Experimental Results." Unfortunately, only medium vacuum conditions of the order of $10^{-9}$ mbar have been obtained during operation at high average RF power to date. A second disadvantage is that the water cooling of the cavity limits the average RF power used for accelerating the bunch charge. This RF power can either be used for obtaining a high accelerating gradient, for operating at high duty cycle, or for a reasonable compromise between both options. In addition, the water cooling system needs to have a high stability and precision in order to keep the gun cavity tuned under different RF power loads. Nevertheless, with proper choice of resonant frequency, cavity and photocathode material as well as sophisticated cavity and cooling channel design, a broad range of average beam currents with high beam quality can be produced, as will be demonstrated in the following subsections.

\section{Superconducting RF Guns}

This photo injector type is schematically very similar to the version previously discussed. The major difference is that the accelerating cavity is superconducting (SC). The low RF losses imply that the cavity can be operated with a high RF duty cycle or in some cases even in CW mode so that a high average beam power can be achieved and the beam energy from the source can be significantly higher than for the DC photocathode gun case. The vacuum condition in the SC gun is good so that NEA cathodes such as GaAs which offer low thermal emittance can be considered an option for normal conducting cathodes in an SC gun. On the other hand, the SC cavity also implies that the space charge compensating solenoid cannot be placed around the cavity itself. One 
possibility to cure this is to use a split injector design, where the solenoid is placed downstream of the gun cavity, either as an SC solenoid or as an NC one downstream of the cold-warm transition from the SC gun cavity. If one wants to produce higher bunch charges with currently available fairly low accelerating gradients at the cathode, it is beneficial when the solenoid is closer to the gun cavity. Another possibility is to improve the focusing within the cavity by applying electrical RF focusing in the cathode region (Janssen and Volkov 2000). Nevertheless, simulations showed that this concept limits the charge per bunch if very good beam quality is required. A newer approach to applying a magnetically focusing RF mode which can overcome this limitation will be presented in section "SC RF Gun Results and Developments at HZDR." For SC RF guns, the photocathode is a more complicated issue than for NC RF guns. It has to be considered if the cathode should be normal conducting or superconducting. In case a NC cathode is chosen, it has to be clarified how the NC cathode can be isolated from the SC cavity and how a highest possible accelerating field can be maintained at the cathode. For the SC cathode case, a cathode material with high quantum efficiency has to be found, and it has to be clarified what average laser power can be deposited on the cathode before it switches to the normal conducting state. One SC RF gun project with its specific solutions will be discussed later in this chapter. More details on superconducting RF technology including SC RF guns are presented in the chapter "Superconducting RF: Enabling Technology for Modern Light Sources" from Jens Knobloch in this book. Compared to the other two basic photo injector types, the SC RF guns are a newer development so that the operating experience for this kind of electron source is increasing but still limited.

\section{High Average Current Electron Sources}

In this section, three photo injector developments providing average beam currents of at least $1 \mathrm{~mA}$ are summarized in Table 2. The projects are described briefly in the following subsections.

\section{Photocathode Gun Development at Cornell}

For the operation of an energy recovery linac-based X-ray source at Cornell, a DC photocathode injector design was published in 2003 (Bazarov and Sinclair 2003). The injector schematic is shown in Fig. 6.

In 2005, an improved design of the Cornell injector was published (Bazarov and Sinclair 2005), reducing the previous design transverse emittance by a factor of about 6 . This impressive improvement in the design was obtained by a multivariate optimization of almost all injector parameters. Twenty-two decision variables had been defined, incorporating all the physical constraints of the problem, e.g., the field strengths and locations of the elements, the RF phases, the transverse and longitudinal laser profiles on the cathode, the bunch charge, and the thermal energy of the electrons leaving the photo cathode. The ASTRA code was used for tracking the electrons and the optimization was run for the two cases of 80 and $800 \mathrm{pC}$ to obtain maximum average brilliance and maximum photon flux per pulse respectively. For details of the optimization procedure and the simulation results, see reference (Bazarov and Sinclair 2005). No single parameter was responsible for the impressive emittance improvement compared to the old design of 2003, but the multivariate optimization changed a large proportion of the design parameters significantly. This study showed the importance and the benefit of a multivariate optimization resulting from the strong correlation 


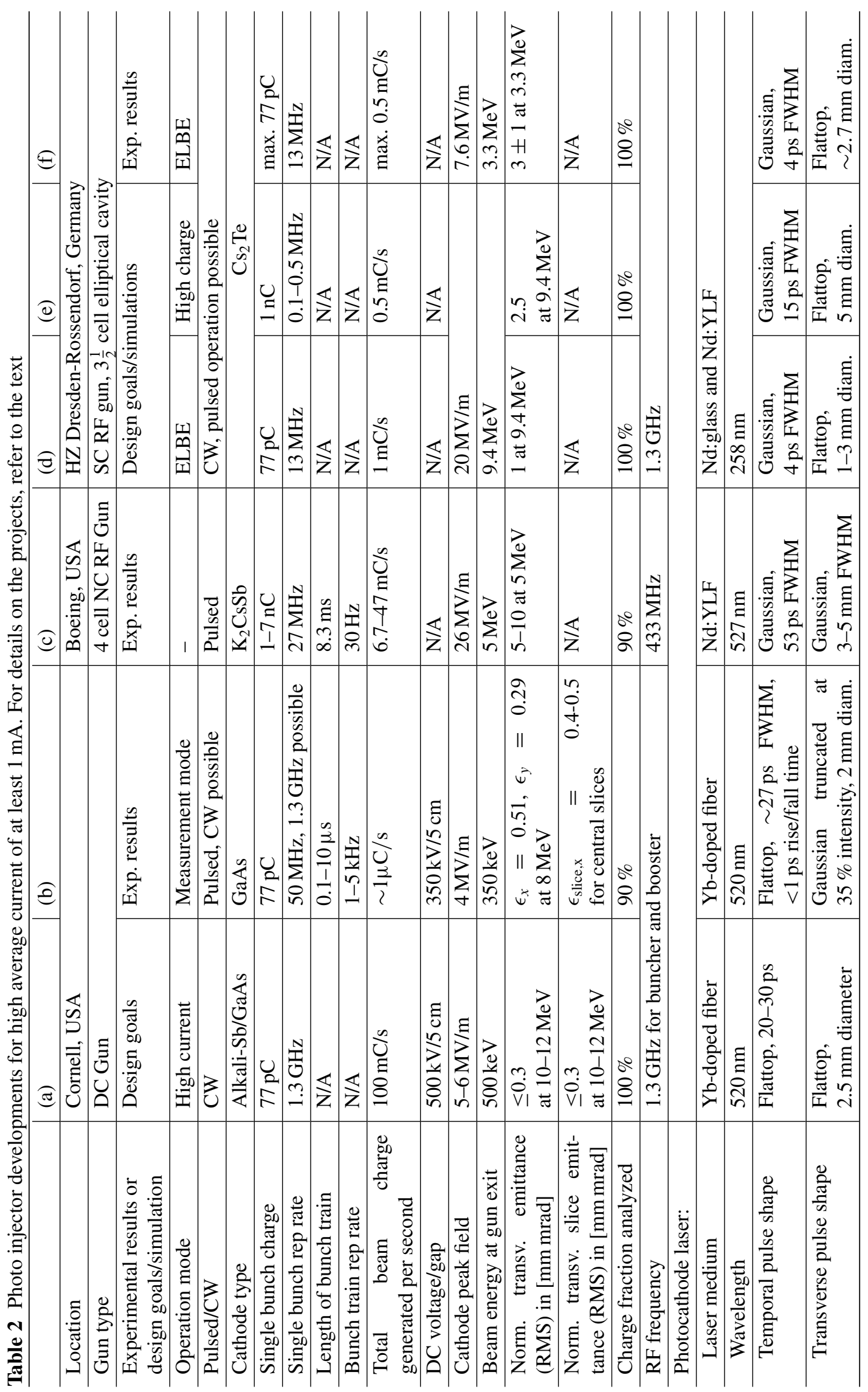




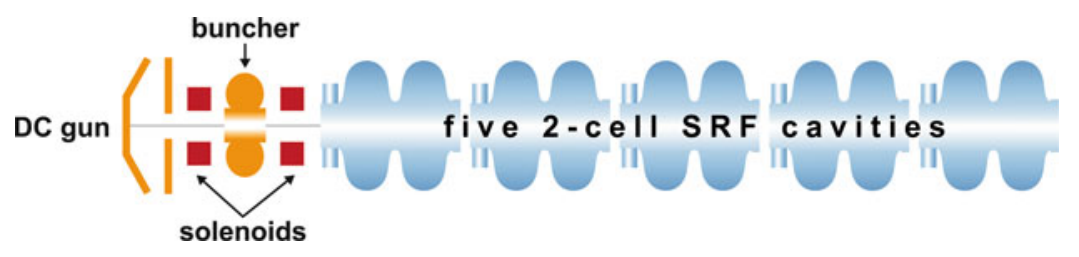

Fig. 6 Schematic of the photoelectron injector for the Cornell ERL-based X-ray source

in the high-dimensional design parameter space. From the simulation results, it is also interesting to note that the optimized transverse laser profiles for both charge cases are truncated elliptical distributions and that the temporal laser profile for the $800 \mathrm{pC}$ case is a flattop profile with short rise/fall time and in the case of $80 \mathrm{pC}$ is a flattop profile with short rise/fall time but with significant depletion in the center of the flattop. These numerically optimized laser shapes are significantly different from the 3D ellipsoidal distributions being discussed in Luiten et al. (2004), LimborgDeprey (2005), and Geer (2005) as well as in sections "Generic Photo Injector Layout and Beam Dynamics Simulations" and "Comparing Photo Injector Performances and Future Trends" in this chapter. Such kind of very useful multivariate optimization later on has also been performed for other photo injector facilities.

The photo injector setup for the ERL-based light source was built and commissioned at Cornell. Different photo cathodes have been tested, and average beam currents of $33 \mathrm{~mA}$ have been shown with a $\mathrm{Cs}_{3} \mathrm{Sb}$ cathode over almost $4 \mathrm{~h}$ and even up to $65 \mathrm{~mA}$ have been demonstrated with a $\mathrm{CsK}_{2} \mathrm{Sb}$ photocathode for a short time (Dunham et al. 2013). This is the highest average beam current ever demonstrated until summer 2013.

Also a detailed beam characterization has been performed at the Cornell photo injector. For operation with a bunch charge of $77 \mathrm{pC}$, a normalized projected transverse emittance of $0.53 \mathrm{~mm}$ mrad was measured for $100 \%$ of the bunch charge (Gulliford et al. 2013). In column (a) of Table 2, the current design parameters of the Cornell photo injector are listed (Dunham, private communication). In addition to the numbers in the table, it must be considered that the RMS bunch length after the injector has to be $\leq 3 \mathrm{ps}$ for the acceleration in the RF cavities downstream (Gulliford et al. 2013). In column (b) of Table 2, some details of the experimental beam characterization with $77 \mathrm{pC}$ bunch charge are given (Gulliford et al. 2013; Dunham, private communication).

Finally, it is interesting to mention that this electron source would be capable of producing polarized electrons with high beam quality if the appropriate photocathode laser and cathodes are used.

\section{NC RF Gun Results from Boeing}

Although the measurements with a $433 \mathrm{MHz}$ normal conducting RF gun at Boeing were performed as long ago as 1992 (Dowell et al. 1993) (see Table 2 (c)), they are still interesting to compare with since they over a long time represented the highest average current produced from a photo injector until the DC gun at Cornell broke the record (see previous section). The gun was operated with long pulse trains at a duty cycle of $25 \%$ with a high average RF power of $600 \mathrm{~kW}$. In spite of the high duty cycle and low RF frequency, the reentrant design of the gun cavity allowed a fairly high peak accelerating field of about $25 \mathrm{MV} / \mathrm{m}$ at the cathode. Since a high accelerating field at the 
cathode is an important ingredient for obtaining a small beam emittance, a reentrant design can be considered an option for future RF guns with high duty cycle; see, e.g., Rimmer (2005).

\section{SC RF Gun Results and Developments at HZDR}

The first beam from a superconducting RF gun was demonstrated in 2002 at the HelmholtzZentrum Dresden-Rossendorf (Janssen et al. 2003). At that time, it was a $1 / 2$ cell SC RF gun with a $\mathrm{Cs}_{2} \mathrm{Te}$ cathode generating up to $20 \mathrm{pC}$ at $26 \mathrm{MHz}$. At a beam energy of $900 \mathrm{keV}$, a normalized projected emittance of $2.5 \mathrm{~mm}$ mrad was measured for $4 \mathrm{pC}$ bunch charge.

After this proof-of-principle experiment, the design of a $3 \frac{1}{2}$ cell SC RF gun began (Janssen 2004). It consists of a specially designed cathode cell and three modified TESLA shape cells. An ASTRA simulation for a $1 \mathrm{nC}$ operation mode with a solenoid outside of the gun cryostat and a subsequent TESLA module with eight SC 9-cell cavities predicted a transverse projected emittance of about $1.7 \mathrm{~mm}$ mrad (Janssen 2004).

For the $3 \frac{1}{2}$ cell cavity, a new idea to improve the space charge compensation process within the SC gun cavity was also developed (Janssen and Volkov 2004; Flöttmann et al. 2004). This idea utilizes an additional magnetically focusing RF mode which is localized in one of the cavity cells. Care was taken that the sum of the surface fields is below the quench limit. Simulations predicted that for a charge of $1 \mathrm{nC}$, a transverse emittance between 0.8 and $1.0 \mathrm{~mm}$ mrad can be obtained (Flöttmann et al. 2004). In Volkov and Janssen (2008), it was shown that for a specially shaped cavity cell, the magnetically focusing RF field is equivalent to the time-independent field of a static solenoid and can be used for the emittance compensation.

Several $3 \frac{1}{2}$ cell SC gun cavities have been produced and put into operation, although not yet exploiting the magnetically focusing RF mode. A particularity of this RF gun is that the cathode is normal conducting, operating at $77 \mathrm{~K}$. It is usually coated with $\mathrm{Cs}_{2} \mathrm{Te}$ and a life time of months has been demonstrated. This ensures high quantum efficiency and relaxes the requirements on the laser system. Several years of operation experience have been gained. Meanwhile the $3 \frac{1}{2}$ cell SC $\mathrm{RF}$ gun is included in the ELBE accelerator and has provided first lasing of an IR FEL driven by an SC RF gun in April 2013 (Teichert et al. 2013). The design parameters for operating the $3 \frac{1}{2}$ cell SC gun at two different beam charges are given in column (d) and (e) of Table 2 (Arnold et al. 2007; Teichert, private communication). One set of experimental results obtained until summer 2013 is listed in column (f) of Table 2 (Teichert, private communication). Further developments on SC gun cavity fabrication and preparation are expected to allow operation at the design peak accelerating cathode gradient of $20 \mathrm{MV} / \mathrm{m}$ which should help to improve the beam quality significantly. For the future, also operation with photocathodes sensitive at the green wavelength like GaAs and $\mathrm{K}_{2} \mathrm{CsSb}$ is foreseen. This would reduce the requirements on the power of the photocathode laser system and allow more head space for and simplify the application of sophisticated photocathode laser pulse shaping techniques. This together with the expected lower thermal emittance from a GaAs photocathode would further improve the beam quality.

\section{Medium Average Current Electron Sources}

In Table 3 two different photo injector developments providing average beam currents between $1 \mu \mathrm{A}$ and $1 \mathrm{~mA}$ are listed. One utilizes fairly long electron bunch trains in a pulsed operation, 
Table 3 Photo injector developments for a medium average current between $1 \mu \mathrm{A}$ and $1 \mathrm{~mA}$. For details on the projects, refer to the text

\begin{tabular}{|c|c|c|c|c|}
\hline & (a) & (b) & (c) & (d) \\
\hline Location & \multicolumn{3}{|c|}{ DESY (PITZ), Germany } & LBNL, USA \\
\hline Gun type & \multicolumn{3}{|c|}{$1 \frac{1}{2}$ cell NC RF gun } & NC RF gun, $\frac{1}{4}$-wave cavity \\
\hline $\begin{array}{lcc}\text { Experimental results } & \text { or } \\
\text { design goals/simulation } & \\
\end{array}$ & $\begin{array}{l}\text { Design goals/ } \\
\text { simulations }\end{array}$ & Exp. results & Exp. results & Exp. results \& simulations \\
\hline Operation mode & Baseline & Baseline & $\begin{array}{l}\text { Lower } \\
\text { charge }\end{array}$ & - \\
\hline Pulsed/CW & \multicolumn{3}{|l|}{ Pulsed } & Pulsed and CW demonstrated \\
\hline Cathode type & \multicolumn{3}{|l|}{$\mathrm{Cs}_{2} \mathrm{Te}$} & $\begin{array}{ll}\text { Testing } & \mathrm{Cs}_{2} \mathrm{Te}, \\
\mathrm{CsK}_{2} \mathrm{Sb} \text { later } & \\
\end{array}$ \\
\hline Single bunch charge & $1 \mathrm{nC}$ & $1 \mathrm{nC}$ & $250 \mathrm{pC}$ & $10 \mathrm{fC}$ to $500 \mathrm{pC}$ demonstrated \\
\hline Single bunch rep rate & $4.5 \mathrm{MHz}$ & \multicolumn{2}{|c|}{$1 \mathrm{MHz}, 4.5 \mathrm{MHz}$ later } & $20 \mathrm{~Hz}$ to $1 \mathrm{MHz}$ \\
\hline Length of bunch train & $600 \mu s$ & \multicolumn{2}{|c|}{$600 \mu \mathrm{s}, \leq 800 \mu \mathrm{s}$ possib. } & N/A \\
\hline Bunch train rep rate & \multicolumn{3}{|l|}{$10 \mathrm{~Hz}$} & \multirow{2}{*}{$\begin{array}{l}\text { N/A } \\
\text { Up to } 300 \mu \mathrm{C} / \mathrm{s} \text { demonstrated, } \\
\text { up to } 1 \mathrm{mC} / \mathrm{s} \text { possible }\end{array}$} \\
\hline $\begin{array}{l}\text { Total beam charge generated } \\
\text { per second }\end{array}$ & $27 \mu \mathrm{C} / \mathrm{s}$ & $6 \mu \mathrm{C} / \mathrm{s}$ & $1.5 \mu \mathrm{C} / \mathrm{s}$ & \\
\hline DC voltage/gap & N/A & N/A & N/A & N/A \\
\hline Cathode peak field & $60 \mathrm{MV} / \mathrm{m}$ & \multicolumn{2}{|l|}{$\sim 60 \mathrm{MV} / \mathrm{m}$} & $\sim 21 \mathrm{MV} / \mathrm{m}$ \\
\hline Beam energy at gun exit & $6.6 \mathrm{MeV}$ & \multicolumn{2}{|l|}{$\sim 6.5 \mathrm{MeV}$} & $800 \mathrm{keV}$ \\
\hline $\begin{array}{l}\text { Norm. transv. emittance } \\
\text { (RMS) in [mm mrad] }\end{array}$ & 0.9 at $\sim 140 \mathrm{MeV}$ & $\begin{array}{l}\epsilon_{x, y}=0.60 \\
\text { at } 25 \mathrm{MeV}\end{array}$ & $\begin{array}{l}\epsilon_{x, y}=0.29 \\
\text { at } 25 \mathrm{MeV}\end{array}$ & $\begin{array}{lll}\text { Simulated: } 0.2 & \text { to } & 0.7 \\
\text { for } 10 \text { to } 300 \mathrm{pC} & & \\
\end{array}$ \\
\hline $\begin{array}{l}\text { Norm. transv. slice emit- } \\
\text { tance (RMS) in [mm mrad] }\end{array}$ & $\begin{array}{l}1.4 \text { for } 1 \mathrm{nC} \\
\text { at } 17.5 \mathrm{GeV}\end{array}$ & N/A & $\mathrm{N} / \mathrm{A}$ & $\begin{array}{llll}\text { Simulated: } 0.1 & \text { to } & 0.6 \\
\text { for } 10 \text { to } 300 \mathrm{pC} & & \\
\end{array}$ \\
\hline Charge fraction analyzed & $100 \%$ & $95 \%$ & $95 \%$ & $95 \%$ \\
\hline RF frequency & \multicolumn{3}{|l|}{$1.3 \mathrm{GHz}$} & $186 \mathrm{MHz}$ \\
\hline \multicolumn{5}{|l|}{ Photocathode laser } \\
\hline Laser medium & \multicolumn{3}{|l|}{ Yb:YAG } & Yb-doped fiber \\
\hline Wavelength & \multicolumn{3}{|l|}{$257 \mathrm{~nm}$} & 266 and $532 \mathrm{~nm}$ available \\
\hline Temporal pulse shape & $\begin{array}{l}\text { Flattop, } \\
2 \text { ps rise/fall time, } \\
20 \text { ps FWHM }\end{array}$ & \multicolumn{2}{|c|}{$\begin{array}{l}\text { Flattop } \\
\leq 2 \mathrm{ps} \text { rise/fall time } \\
\sim 22 \mathrm{ps} \text { FWHM }\end{array}$} & $\begin{array}{l}\text { Flattop, } \\
\sim 1 \text { ps rise/fall } \\
50 \text { ps FWHM }\end{array}$ \\
\hline Transverse pulse shape & $\begin{array}{l}\text { Flattop, } \\
0.53 \mathrm{~mm} \text { RMS }\end{array}$ & $\begin{array}{l}\sim \text { Flattop, } \\
\sim 0.3 \mathrm{~mm} \\
\text { RMS }\end{array}$ & $\begin{array}{l}\sim \text { Flattop, } \\
\sim 0.18 \mathrm{~mm} \\
\text { RMS }\end{array}$ & $\begin{array}{l}\text { Gaussian, } 0.05-0.5 \mathrm{~mm} \text {, trun- } \\
\text { cation possible }\end{array}$ \\
\hline
\end{tabular}

while the other operates in $\mathrm{CW}$ mode. The projects are described in the following subsections. The description of the PITZ photo injector setup at DESY, Zeuthen site, is done in more detail to serve as an example for the components required to generate high brightness beams and diagnostics tools needed to control the photo injector input parameters and to perform detailed phase-space characterizations. Also the experimental optimization procedure is described in more detail since it shows a good example of the interdependencies between the different photo injector parameters. In addition, the comparison of experimental data and simulations shows that the experimental optimization is necessary since the beam dynamics simulations do not predict the interdependencies correctly in all details for highly space-charge dominated beam parameters. The experimental results on the normalized projected transverse emittance achieved at PITZ 
are shown in section "Experimental Results" and represent the best results currently obtained worldwide for a wide range of bunch charges from 0.02 to $2 \mathrm{nC}$.

\section{NC RF Gun Results at PITZ}

The photo injector test facility at DESY, Zeuthen side (PITZ), was built to develop and experimentally optimize high brightness photoelectron sources for the Free electron LASer in Hamburg (FLASH) and the European X-ray Free Electron Laser (European XFEL). Both FEL facilities have superconducting linacs so that the important features of the source development at PITZ are (a) the generation of long electron bunch trains to have an efficient use of the pulsed superconducting RF of the linacs and (b) the stringent requirements on the transverse emittance in order to allow saturation of the short wavelength FELs after minimum traveling paths in the corresponding undulators. Since the most challenging requirements on beam quality come from the European XFEL, their specs are listed in column (a) of Table 3 (Ferrario et al. 2001; The European X-Ray Free-Electron Laser 2007). The original requirement for the photo injector to deliver a normalized projected emittance of $0.9 \mathrm{~mm} \mathrm{mrad}$ for $1 \mathrm{nC}$ bunch charge (Ferrario et al. 2001) has meanwhile been under-matched by the experimental results found at PITZ (see column (b) of Table 3; the discussion of the data is given in section "Experimental Results"). Together with the additional finding at LCLS that the slice emittance degradation from the photo injector to the undulator is fairly small, this resulted in a new parameter set foreseen for the operation of the European XFEL: The slice emittance for $1 \mathrm{nC}$ bunch charge at the XFEL undulator is now expected to be $1.0 \mathrm{~mm}$ mrad (Decking and Limberg 2013) while before $1.4 \mathrm{~mm} \mathrm{mrad}$ had been expected (The European X-Ray Free-Electron Laser 2007). This will allow a further extension of the FEL capabilities (e.g., shorter wavelength, higher intensities) for the users.

As an example for the general experimental setup of a photo injector test facility, the current PITZ layout is described in the following subsection. Other test facilities have similar diagnostics components. The emittance diagnostics, the experimental optimization procedure, and the exceptionally good emittance data measured at PITZ are discussed in the subsequent subsections.

\section{Layout of the PITZ Facility}

A general schematic diagram of the PITZ setup is shown in Fig. 7. The early developments of the accelerator layout and details of the latest installation are given in references Stephan et al. (2010) and Krasilnikov et al. (2012), respectively. Here only the current version of the main components is described. The accelerator consists of a photocathode RF gun, a normal conducting booster cavity, and various systems for cathode laser and electron beam diagnostics.

The PITZ RF gun is a 1.6 cell L-band normal conducting copper cavity with a $\mathrm{Cs}_{2} \mathrm{Te}$ photo cathode (Dwersteg et al. 1997). A load-lock system allows an easy exchange of the photocathodes without breaking the gun vacuum. The gun cavity is supplied with main and bucking solenoids for control and mitigation of space charge forces in electron bunches of very high density. The RF feed of the gun is realized using a $10 \mathrm{MW}$ multi-beam klystron which supplies the power via two waveguide arms. A T-combiner is installed in the vicinity of the RF gun and serves to superpose the waves from these two arms to feed the gun cavity. A phase shifter in one of the waveguides is used to match the amplitudes and phases of the mixing waves. The combined wave is fed through 

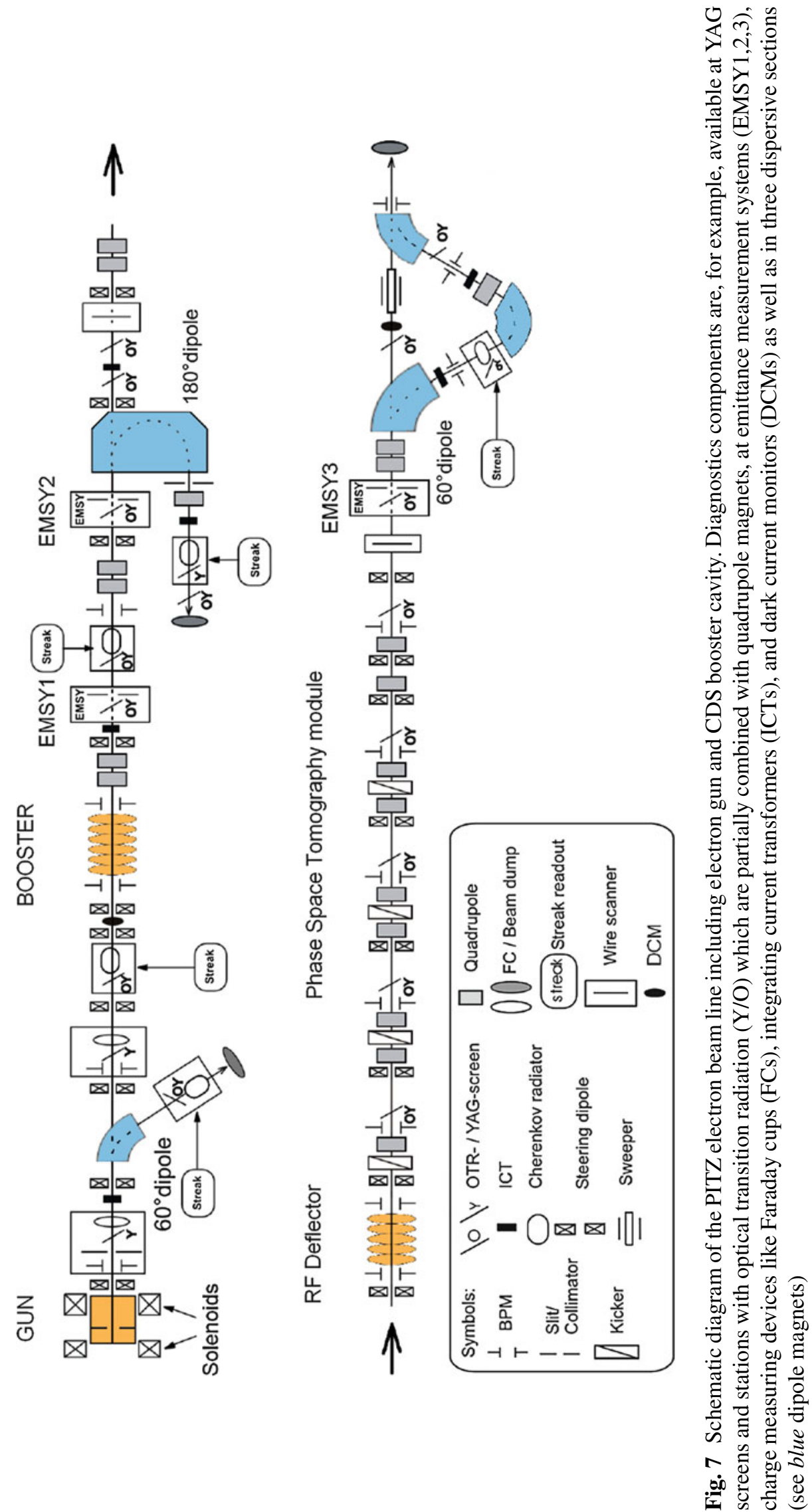
a rotationally symmetric coaxial coupler into the gun. The RF feed regulation is based on signals measured from the forward wave after the T-combiner and the wave reflected from the gun cavity. The shot-to-shot RMS phase jitter is $\sim 0.1 \mathrm{deg}$; the phase slope within the RF pulse is zero within measurement precision.

The PITZ gun cavity is typically conditioned to a peak power of $\sim 6 \mathrm{MW}$. The maximum RF pulse duration is usually $700 \mu \mathrm{s}$ at $10 \mathrm{~Hz}$ repetition rate. The typical RF pulse length used during emittance studies was up to $300 \mu \mathrm{s}$. While the RF conditioning of the gun cavity is usually performed with a Molybdenum cathode plug, a $\mathrm{Cs}_{2} \mathrm{Te}$ cathode is inserted in the cavity for beam operation without breaking the vacuum with help of the cathode load-lock system. Since 2007 the dry-ice sublimation impulse cleaning procedure has been applied to all gun cavities before the RF conditioning at PITZ (Stephan et al. 2008). This resulted in a significant reduction of the dark current. The cavity prototypes \#3.1 and \#4.3 that have been conditioned at PITZ showed a dark current of 15 and $50 \mu \mathrm{A}$ at $\sim 6 \mathrm{MW}$ peak power, respectively (Isaev et al. 2013). Such a low dark current is very important for operating a superconducting linac. At the same time, it improves the quality of electron beam measurements due to a reduced background signal.

The photocathode laser system was developed by the Max-Born Institute, Berlin (Will et al. 2005; Will and Klemz 2008). Different active laser materials have been tested and the current setup is described in the following. The Yb:YAG-based pulse train oscillator is capable of generating pulse trains with up to 800 micropulses and $1 \mu$ s spacing between the pulses within the train. The trains are usually produced at $10 \mathrm{~Hz}$ repetition rate. An Yb:YAG regenerative amplifier and a two-stage Yb:YAG booster amplifier together with frequency conversion crystals provide UV output pulses with a wavelength of $257 \mathrm{~nm}$ and a maximum energy of $\sim 10 \mu \mathrm{J}$ per micropulse. A temporal pulse shaper based on 13 birefringent crystals is used to transform the initial short Gaussian pulse into a flattop profile with rise and fall times as short as $\sim 2$ ps and a pulse duration of 21-22 ps FWHM (Will and Klemz 2008). An optical sampling system (OSS) based on an optical cross-correlation technique is used at PITZ to measure the temporal profile of the UV output pulses with a resolution of better than 1 ps. A typical temporal profile measured with the OSS is shown in Fig. 8, left plot. Transverse pulse shaping is realized by cutting out the central part of the laser spot with a beam shaping aperture (BSA) and its consequent imaging onto the photo cathode. Several plates with fixed sets of BSAs were installed in the laser beam line. This corresponded to discrete values of the laser spot diameter used during the emittance optimization for various bunch charges in the 2011 run presented in section "Experimental Results." Now a BSA with remotely variable diameter is installed in the PITZ optical beam line. The transverse distribution of the cathode laser pulses is monitored with a UV-sensitive CCD camera placed at a location which is optically equivalent to the real cathode position. A typical transverse distribution is shown in the right plot of Fig. 8. The cathode laser pulses are sent to the photocathode by means of a vacuum mirror installed slightly off the beam axis in a diagnostic cross $0.72 \mathrm{~m}$ downstream of the cathode plane.

A booster cavity based on a cut disk structure (CDS) developed by INR (Moscow) (Paramonov et al. 2010) provides a typical final beam momentum of $24.5 \mathrm{MeV} / \mathrm{c}$. The CDS booster was specially designed for PITZ to allow maximum momentum gain with limited RF power and supports long pulse train operation.

Many different devices for beam diagnostics are installed along the PITZ beamline; see Fig. 7. Besides the emittance diagnostics which is described in the next subsection, there are three spectrometer systems with dispersive sections (see blue dipole magnets in Fig. 7) to measure the longitudinal momentum and in addition the longitudinal phase space using a streak 

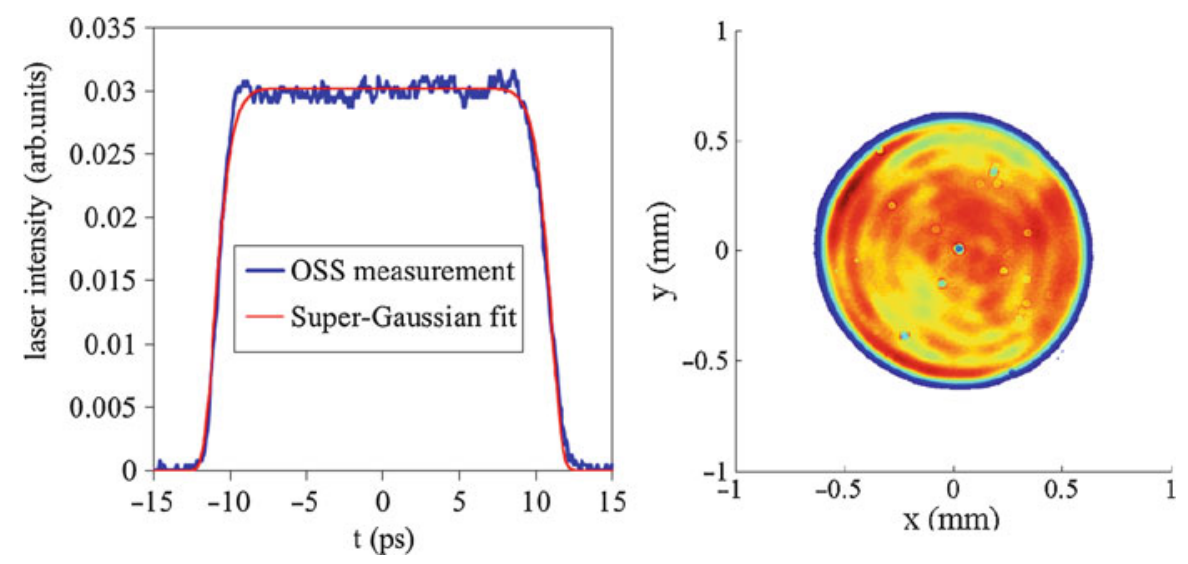

Fig. 8 Photocathode laser pulse temporal profile (left plot) and transverse distribution (right plot). The temporal profile measured with the OSS is also supplied with the Super-Gaussian fit curve: $F(t)=\exp \left[-\frac{1}{2}\left(\frac{t}{10.627}\right)^{16.679}\right]$

camera readout system (Rönsch 2009). A transverse deflecting structure designed and built by INR (Moscow) (Kravchuk et al. 2010) is installed but still has to be commissioned. It is expected to allow longitudinal phase-space measurements with a time resolution of $0.3 \mathrm{ps}$ (Malyutin et al. 2012). The bunch charge is measured using integrating current transformers (ICTs) for a range of $0.2-2 \mathrm{nC}$; lower charges $(\leq 0.2 \mathrm{nC})$ are detected using the Faraday cup (FC) in the first diagnostic cross $(\sim 0.8 \mathrm{~m}$ from the cathode plane). Many YAG powder and OTR screens are distributed over the beamline and are read out with 12-bit CCD cameras to measure the transverse distribution of the electron beam. Wire scanners are installed to monitor the bunch profile when operating with long bunch trains. Beam position monitors (BPMs) are used to monitor the trajectory of the beam. Quadrupoles are distributed over the beam line to focus the beam and match it to the tomography section, where a transverse phase-space tomography can be performed (Asova 2012). Many steerer magnets are used to control the beam trajectory. A sweeper is installed in front of the beam dump to prevent damage generated by the high brightness beams.

\section{Emittance Diagnostics}

The transverse phase space of the electron beam is measured downstream of the booster either by one of the three slit-scan stations (Staykov 2009; Vashchenko 2013) or by using the tomography module. One important advantage of the tomography module is that it measures the phase space of both transverse planes simultaneously and is also sensitive at low bunch charges. On the other hand, the space charge forces of the entire high brightness beam are still very strong at PITZ due to the relatively low beam energy. This means that space charge effects have to be taken into account for the beam matching into the tomography module as well as for the analysis of the experimental data (the phase advance inside the tomography module is changed by the space charge effects). Therefore, the tomography module is used for specific measurements, but the three emittance measurement systems based on the slit-scan technique and located at 5.74, 7.13, and $16.19 \mathrm{~m}$ from the photo cathode are used for standard machine parameter scans (see EMSY1, EMSY2, EMSY3 in Fig. 7). The experimental emittance optimization and the results presented in section "Experimental Results" were obtained with the station EMSY1. The emittance measurement system contains horizontal and vertical actuators supplied with YAG powder and optical transition radiation screens as well as slit masks with $10 \mu \mathrm{m}$ opening made of $1 \mathrm{~mm}$ 
thick tungsten. The slit mask converts a space-charge dominated electron beam into an emittancedominated beamlet which is characterized with a screen $2.64 \mathrm{~m}$ downstream of the slit. The part of the beam which is hitting the mask is scattered and builds a homogeneous background. By moving the slit mask transversely over the beam spot, one obtains local divergence profiles of the electron beam. The transverse phase space $\left(x, x^{\prime}\right)$ can be reconstructed from the whole set of slitscan data by correlating the measured local divergence profiles with the corresponding slit mask positions. The YAG screen at the EMSY position is used to measure the RMS size of the whole beam $\sigma_{x}$. This value is typically larger than the RMS size of the phase-space distribution purely obtained from the slit scan $\sqrt{\left\langle x^{2}\right\rangle}$ due to the limited sensitivity for very low-intensity beamlets at the tails of the phase-space distribution. With the assumption that the measured RMS divergence after removing the linear covariance $\left\langle x x^{\prime}\right\rangle$ from the measured phase space is close to the real uncorrelated divergence, the expression for the RMS normalized (horizontal) emittance takes the form (Krasilnikov et al. (2012) and Rimjaem et al. (2012))

$$
\epsilon_{n, x}=\beta \gamma \frac{\sigma_{x}}{\sqrt{\left\langle x^{2}\right\rangle}} \sqrt{\left\langle x^{2}\right\rangle\left\langle x^{\prime 2}\right\rangle-\left\langle x x^{\prime}\right\rangle^{2}}
$$

where the factor $\beta \gamma=\sqrt{\gamma^{2}-1}$ can be calculated from the relativistic Lorentz factor $\gamma$ which is determined from the measurement of the longitudinal momentum of the electron beam. It should be noticed that applying the correction factor $\frac{\sigma_{x}}{\sqrt{\left\langle x^{2}\right\rangle}}$ which usually takes values between 1.1 and 1.3 is a conservative approach to take into account the full beam distribution including the beam tails. This allows to calculate RMS emittance quantities including $100 \%$ of the generated beam charge (100\% RMS emittance values). More details on the emittance measurement procedure at PITZ can be found in Krasilnikov et al. (2012) and Rimjaem et al. (2012).

In order to use the full dynamic range of the 12-bit camera for reading the beam or beamlet image, the maximum intensity of the image is adjusted to have pixels with values of at least $\sim 3,000$ but still the images must not be saturated (the saturation level is $2^{12}-1=4,095$ ). This intensity criterion (" 3,000 criterion") is achieved by an interplay of the camera gain and the number of pulses in a train while keeping the number of pulses as low as possible to minimize the influence of beam jitter.

\section{Experimental Optimization Procedure}

The experimental procedure for minimizing the projected normalized transverse emittance for a given bunch charge consists of a set of consequent steps (Krasilnikov et al. 2012).

1. Laser temporal profile - The temporal profile of the cathode laser pulse is checked using the OSS. The goal shape is a zero-sloped flattop distribution with the shortest possible rise and fall time and smallest intensity modulations. By tuning the corresponding crystals in the pulse shaper, it is possible to obtain a flattop profile as shown in Fig. 8, left plot.

2. Laser transverse distribution - As described in section "Layout of the PITZ Facility" a BSA is inserted in the laser beamline and the resulting transverse shape is monitored by a UV-sensitive CCD camera. The aim is a radial homogeneous distribution. The optimization is realized by a transverse displacement of the BSA to the position within the incident laser beam with the highest integral homogeneity. This is followed by the beam-based alignment of the laser on the photocathode (Krasilnikov et al. 2005). 
3. RF gun phase - The RF gun phase is adjusted w.r.t. the phase of maximum mean momentum gain (MMMG). The momentum measurements in the low-energy dispersive arm (DISP1 in Fig. 7) as a function of the gun launch phase are done using 1 deg steps with already tuned RF feedback in the gun cavity. Because of flatness of the mean momentum dependence on the gun phase, the MMMG phase is defined with an accuracy of about $0.5-1 \mathrm{deg}$. Then the desired offset from the MMMG phase is set, and the momentum distribution is measured for this working phase (i.e., for the gun phase to be used for the emittance measurements). A scanning step of $3 \mathrm{deg}$ has typically been used for the emittance optimization as a function of the gun phase. The laser intensity fine-tuning to produce the given bunch charge for the adjusted gun phase is completing this step of the optimization procedure.

4. Booster phase - The booster phase is also adjusted w.r.t. the MMMG phase. Beam momentum measurements in the first high-energy dispersive arm (DISP2 in Fig. 7) as a function of the booster phase are used to find the booster MMMG phase. It should be noticed that the RF pulse duration in the gun and in the booster is adjusted to support the electron bunch train length needed for high-quality emittance measurements; typically up to $300 \mu \mathrm{s}$ long RF pulses are used. Although the booster gradient (the RF peak power in the cavity) is usually kept constant during one sequence of emittance measurements, this is another tuning parameter to match the electron beam dynamics to the booster acceleration and further improve the emittance conservation process as mentioned in section "Generic Photo Injector Layout and Beam Dynamics Simulations."

5. Emittance measurements versus main solenoid current - Emittance measurements as a function of the main solenoid current are performed. The emittance measurement procedure is roughly described in section "Emittance Diagnostics," more details can be found in Section IV.A.5. of Krasilnikov et al. (2012). Each value of the main solenoid current is set through the maximum value $(500 \mathrm{~A})$ in order to prevent the hysteresis ambiguity, so the solenoid scan is performed from higher to lower values in the scan range. The bucking solenoid is tuned each time to compensate the longitudinal magnetic field at the cathode. A typical step width of 1-2 A is used for the main solenoid scan. For each solenoid current, both $\mathrm{x}$ and $\mathrm{y}$ emittances are being measured consequently.

6. Statistical measurements for the best point - For the solenoid current $I_{\text {main }}^{*}$ delivering the smallest value of the geometrical mean $\sqrt{\epsilon_{n, x} \cdot \epsilon_{n, y}}$, statistical measurements of the emittance are taken (for details, see Section IV.A.6. of Krasilnikov et al. 2012). This reproducibility check aims to control the stability of the obtained emittance minimum.

To optimize the emittance compensation process for a space charge-dominated beam as discussed in section "Generic Photo Injector Layout and Beam Dynamics Simulations," the main parameters at PITZ are the appropriate choice of the laser profiles (transversely and longitudinally), a high peak accelerating gradient of $60 \mathrm{MV} / \mathrm{m}$ at the cathode and the tuning of the main solenoid current. Obviously not all parameters that previously had been optimized in simulations can be realized in practice, e.g., limitations on the length of the laser pulse rise and fall time, limitations on the transverse homogeneity of the photocathode laser profile on the cathode, mechanical constraints on the solenoid, and booster position. Also the choice of specific baseline goal parameters (e.g., operation at a bunch charge of $1 \mathrm{nC}$ ) might limit the capabilities for other goal parameter sets (e.g., operation at lower bunch charge $\rightarrow$ possible limitations due to sensitivity of diagnostics). Therefore, a careful choice of the desired operation parameters of the photo injector 
is required, and since the numerical optimization is expected to overestimate the final beam quality, sufficient head space has to be foreseen for the practical realization.

\section{Experimental Results}

The general experimental procedure for the minimization of the transverse projected emittance at PITZ as described in detail in section "Experimental Optimization Procedure" was applied for bunch charges in the range from 0.02 to $2 \mathrm{nC}$. For each level of the bunch charge, several laser spot sizes (BSAs) were tested to deliver the smallest emittance. Also the RF gun launch phase was varied for higher bunch charges of 1 and $2 \mathrm{nC}$. For each point (BSA, gun phase), the main solenoid current was scanned to find its optimum value, yielding the minimum value of the geometrical mean of the measured RMS normalized emittance in both transverse planes. The gun gradient was fixed to the maximum available at that moment (a peak electric field at the cathode of $\sim 60.6 \mathrm{MV} / \mathrm{m}$ ) yielding a maximum mean momentum of the electron beam of $\sim 6.7 \mathrm{MeV} / \mathrm{c}$ measured downstream of the gun in the low-energy dispersive arm. The CDS booster was operated at the maximum acceleration phase at a peak field, yielding a maximum mean momentum of the electron beam of $\sim 25 \mathrm{MeV} / \mathrm{c}$.

The measured projected $x y$-emittance $\left(\sqrt{\varepsilon_{x} \varepsilon_{y}}\right)$ as a function of the RMS size of the laser spot at the cathode is shown in Fig. 9 for various bunch charges together with simulated curves. All simulated curves are obtained from a laser spot size variation around the optimum value, while the other machine parameters were fixed to their optimum values in the simulations. One can clearly see a rather large discrepancy in the optimum laser spot size for higher bunch charges $(2 \mathrm{nC}, 1 \mathrm{nC}$, and $0.25 \mathrm{nC}$ ). This discrepancy becomes smaller with decreasing charge resulting in a quite good agreement for $0.1 \mathrm{nC}$. These discrepancies are clearly related to space charge effects in the vicinity of the photo cathode, and they have already been observed by simulations of the photo emission

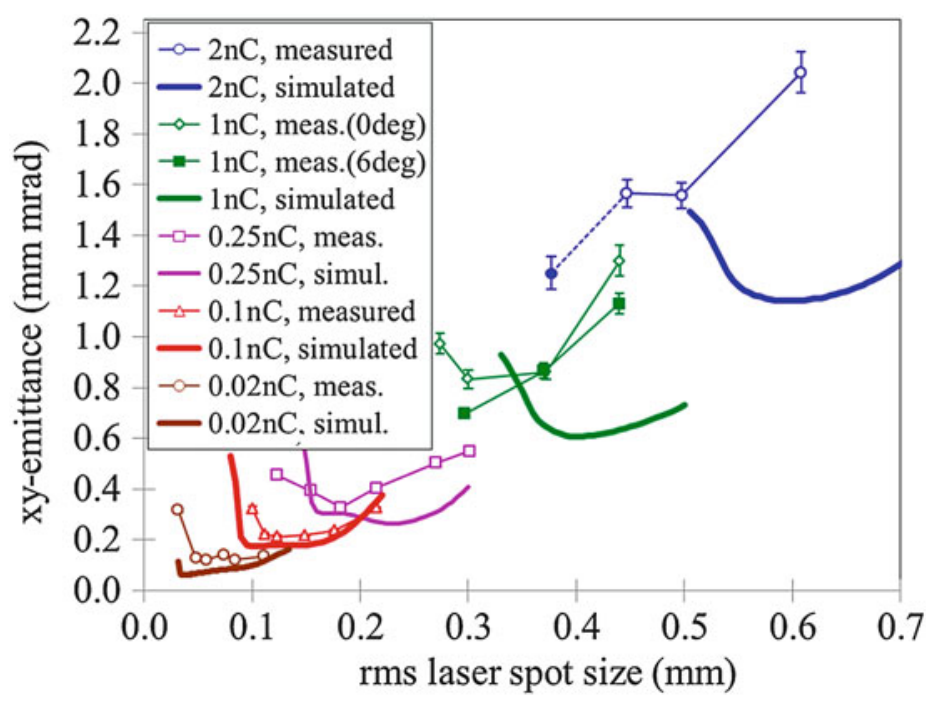

Fig. 9 Measured and simulated RMS normalized emittance as a function of the laser RMS spot size at the cathode for various bunch charges. The two experimental curves for $1 \mathrm{nC}$ correspond to different gun phases. Empty markers of the $2 \mathrm{nC}$ curve indicate gun operation at MMMG phase, whereas the filled marker shows the emittance value measured for +6 deg gun phase. The statistical error bars corresponding to item 6 in section "Experimental Optimization Procedure" are shown 


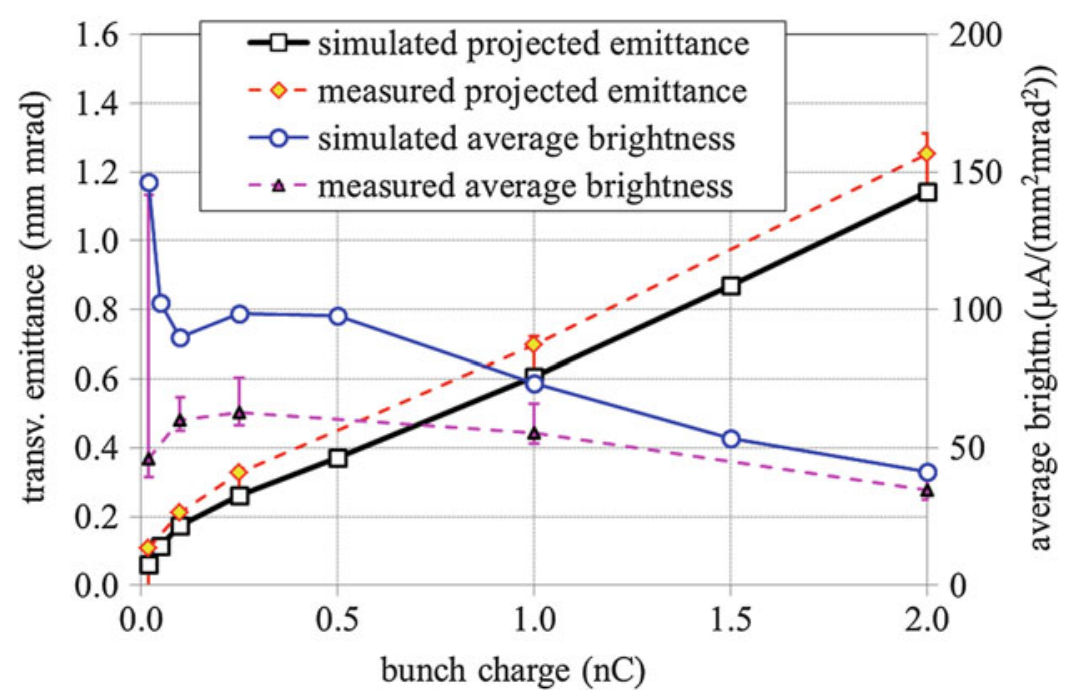

Fig. 10 Measured and simulated RMS normalized transverse emittance as a function of the bunch charge. The measured and simulated average brightness calculated according to Eq. 4 is plotted at the right axis

of the electron bunch with high space charge density at the cathode. More detailed studies on this subject can be found in Krasilnikov et al. (2012).

The measured minimum emittance results are shown in Fig. 10 as a function of the bunch charge together with the corresponding simulated data (minimum emittance values shown in Fig.9). The average brightness of the injector calculated using Eq. 4 for the simulated and measured data is plotted as well. The duty cycle (27,000 electron bunches per second) of the European XFEL injector was applied for these calculations. Besides statistical errors, systematic errors (see Vashchenko 2013) are also included in this plot. There is a general agreement in the measured and simulated emittance dependencies. The simulated brightness has a local maximum between 0.25 and $0.5 \mathrm{nC}$ and a local minimum around $0.1 \mathrm{nC}$, whereas the measured brightness has a maximum around $0.25 \mathrm{nC}$. One should notice that for the simulations as well as for the measurements, the cathode laser pulse duration was fixed at $21.5 \mathrm{ps}$ (FWHM). This implies a significant modification of the space charge effect during emission from the cathode when the bunch charge is increased. Thus, estimated from the simulations, the aspect ratio $\frac{c \sigma_{t}}{\sigma_{x, y}}$ of the optimum laser pulse (or initial electron bunch) is $\sim 50$ for $0.02 \mathrm{nC}$ whereas this ratio is $\sim 3$ for the case of $2 \mathrm{nC}$. The reduction of the emittance with decreased bunch charge is stronger than the corresponding current reduction, and significantly higher average slice brightness was simulated for low charges. The dependence of the measured brightness at lower bunch charges can be explained by rather large measurement errors due to systematic limitations of the measurement setup, especially small beam and beamlet sizes and increased relative position jitter of the electron beam at the position of the slit mask lead to an overestimation of the emittance.

The emittance measurement procedure at PITZ is supposed to be as conservative as possible (see also section "Emittance Diagnostics"). This means that as much as possible, electron beamrelated signal is collected and involved in the phase space reconstruction. The emittance values are obtained from these measurements without any assumption concerning the particle distribution function. This approach results in a conservative estimation of the RMS emittance values and the phase-space distributions, which contain a significant part of beam particles far distant from the phase space center. Those outlying particles are not expected to play a significant role in the later 

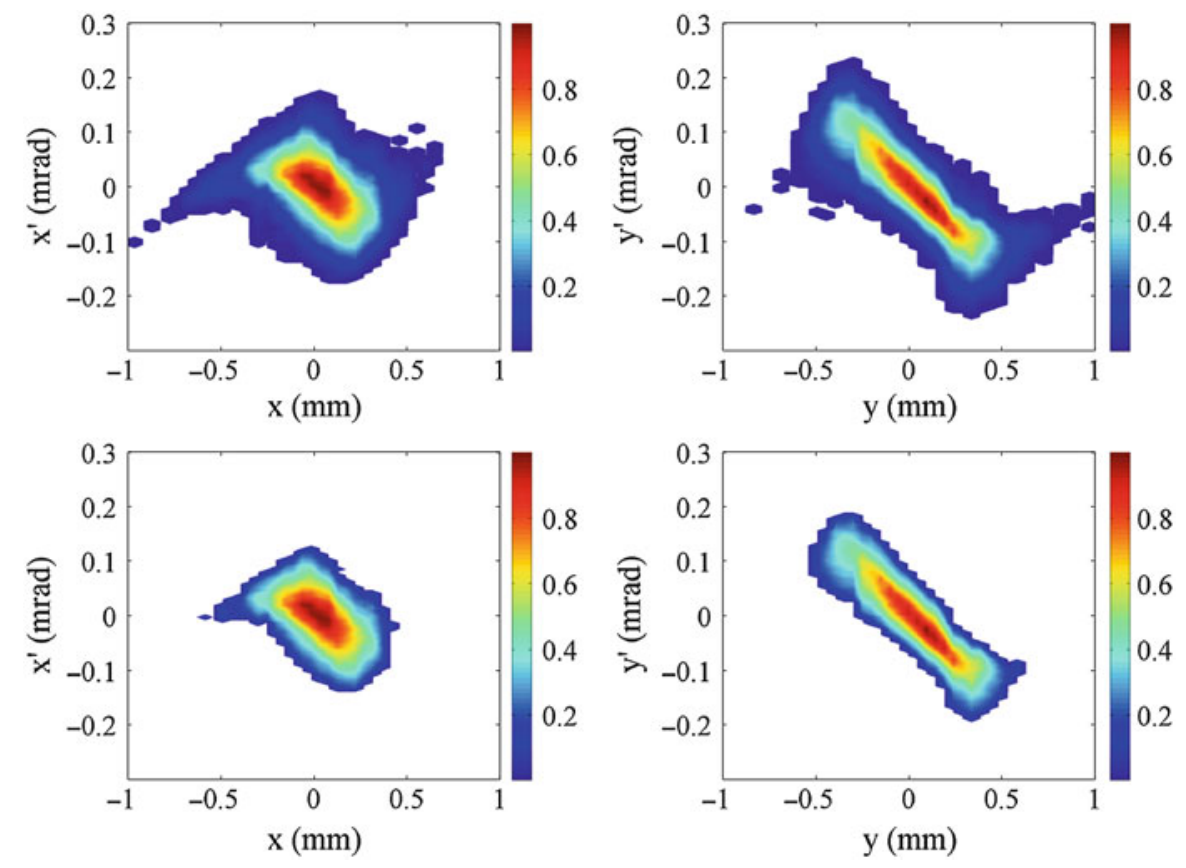

Fig. 11 Measured horizontal (left column) and vertical (right column) phase space for a bunch charge of $1 \mathrm{nC}$. The upper row corresponds to the raw data (100\% of the measured beam signal), whereas the bottom plots are obtained for $90 \%$ of the measured charge originating from those areas of the phase space where the intensity is above a given threshold

FEL lasing process but have a substantial impact on the emittance values. So, one can apply a charge cut procedure to the measured raw phase spaces in order to study the structure of the core emittance and the role of the beam halo. The charge cut is performed on the measured phasespace distributions by discriminating phase-space areas with intensities lower than an introduced threshold. Such a charge cut applied at PITZ is a well-defined, controlled, consistent procedure to remove off-core particles from the analysis. In contrary, alternative methods like Gaussian fits or truncating the distributions from beam projections during quadrupole scan procedures cannot prevent to neglect phase-space regions in some of the projections and still include them in others which makes the analysis not fully consistent. The PITZ charge cut procedure is illustrated by Fig. 11, where the transverse phase spaces experimentally optimized for the case of $1 \mathrm{nC}$ electron beam charge are shown. The upper row of plots shows the raw-measured horizontal and vertical phase spaces (no charge cut performed). The normalized RMS emittance values for this case are $0.707 \pm 0.032 \mathrm{~mm} \mathrm{mrad}$ and $0.685 \pm 0.024 \mathrm{~mm}$ mrad for the $x$-and $y$-planes, respectively. The quoted errors are statistical uncertainty only (see item 6 in section "Experimental Optimization Procedure"). The bottom row of plots shows the results for a $10 \%$ charge cut applied to the raw phase spaces. The corresponding emittance values are $0.543 \pm 0.025 \mathrm{~mm} \mathrm{mrad}$, and $0.515 \pm 0.017 \mathrm{~mm} \mathrm{mrad}$, respectively.

The resulting core emittance measured for various bunch charges is shown in Fig. 12. The charge cut procedure was applied to the corresponding measured optimum $x$ - and $y$-phase spaces for each bunch charge case, and then the geometric mean of both emittance values was calculated as a function of the charge cut. As expected, the strongest reduction of the transverse emittance is obtained by neglecting the low-intensity signal far outside of the core phase-space distribution. This also means that for comparing emittance numbers, the details of the emittance analysis 


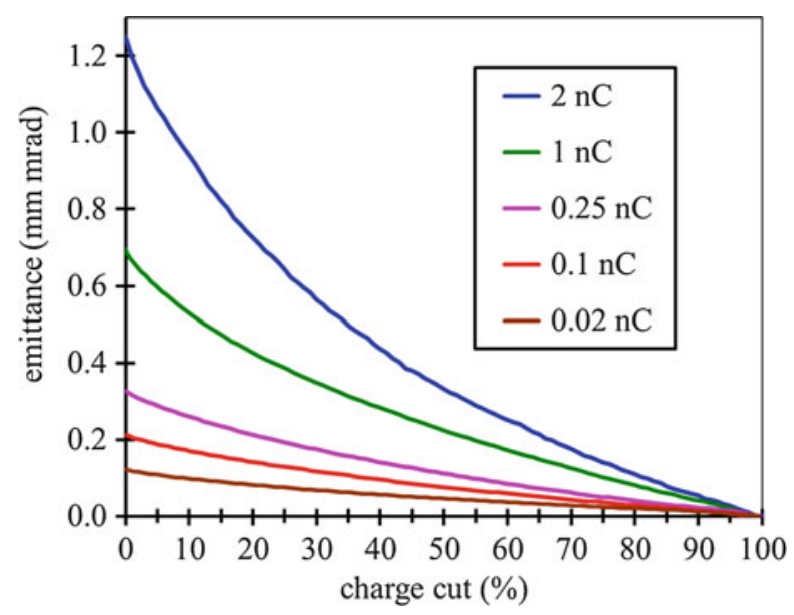

Fig. 12 Measured core $x y$-emittance for different charges as a function of the charge cut. A charge cut of $0 \%$ corresponds to the emittance values from the raw phase space with full beam signal taken into account

Table 4 Experimentally optimized photo injector parameters. For details, see the text

\begin{tabular}{|c|c|c|c|c|c|c|}
\hline \multirow{2}{*}{$\begin{array}{l}\text { Bunch } \\
\text { charge } \\
(\mathrm{nC})\end{array}$} & \multirow{2}{*}{$\begin{array}{l}\text { Gun } \\
\text { phase } \\
\text { (deg) }\end{array}$} & \multirow{2}{*}{$\begin{array}{l}\text { Main } \\
\text { solenoid } \\
\text { current (A) }\end{array}$} & \multirow{2}{*}{$\begin{array}{l}\text { Laser } \\
\text { RMS spot } \\
\text { size (mm) }\end{array}$} & \multicolumn{3}{|c|}{ Measured normalized projected emittance } \\
\hline & & & & $\begin{array}{l}\varepsilon_{x y}(100 \%) \\
\mathrm{mm} \mathrm{mrad}\end{array}$ & $\begin{array}{l}\varepsilon_{x y}(95 \%) \\
\mathrm{mm} \mathrm{mrad}\end{array}$ & $\begin{array}{l}\varepsilon_{x y}(90 \%) \\
\mathrm{mm} \mathrm{mrad}\end{array}$ \\
\hline 2.0 & 6 & 395 & 0.377 & $1.251 \pm 0.064$ & $1.064 \pm 0.054$ & $0.939 \pm 0.048$ \\
\hline 1.0 & 6 & 396 & 0.296 & $0.696 \pm 0.020$ & $0.596 \pm 0.017$ & $0.529 \pm 0.015$ \\
\hline 0.25 & 0 & 393 & 0.182 & $0.328 \pm 0.010$ & $0.289 \pm 0.009$ & $0.260 \pm 0.008$ \\
\hline 0.10 & 0 & 394 & 0.123 & $0.212 \pm 0.006$ & $0.188 \pm 0.006$ & $0.170 \pm 0.006$ \\
\hline 0.02 & 0 & 388 & 0.085 & $0.121 \pm 0.001$ & $0.108 \pm 0.001$ & $0.098 \pm 0.001$ \\
\hline
\end{tabular}

procedure, e.g., neglecting particles outside the beam core by fitting Gaussian distributions to measured beam projections or including all particle in the reconstruction, can have a major influence on the emittance results obtained.

The main results of the experimental optimization of the high brightness photo injector at PITZ are summarized in Table 4. Besides optimum photo injector parameters (RF gun launch phase w.r.t. to the MMMG, the main solenoid current, and the laser spot size $\sigma_{x y}^{l}$ at the cathode), the measured minimum projected normalized emittance and corresponding 95 and $90 \%$ core emittance values are given for different charges. Only statistical emittance errors are shown in the table, which for the 95 and $90 \%$ core emittance data are obtained by applying the charge cut procedure to the different phase spaces measured during the statistical measurements as explained in section "Experimental Optimization Procedure," item 6, and calculating the statistical uncertainty from those results. The total systematic uncertainty of the emittance results is around $10 \%$ for bunch charges between $100 \mathrm{pC}$ and $2 \mathrm{nC}$ and reaches about $150 \%$ overestimation for a bunch charge of $20 \mathrm{pC}$ due to limitations of the optical readout system (Vashchenko 2013).

\section{NC RF Gun Development for CW Operation at LBNL}

Another very interesting example for a photoelectron source with the capability to generate fairly high average current is the Advanced Photoinjector Experiment (APEX) at the Berkeley National Laboratory (Staples et al. 2006; Baptiste et al. 2009; Sannibale et al. 2012). Driving the success of 


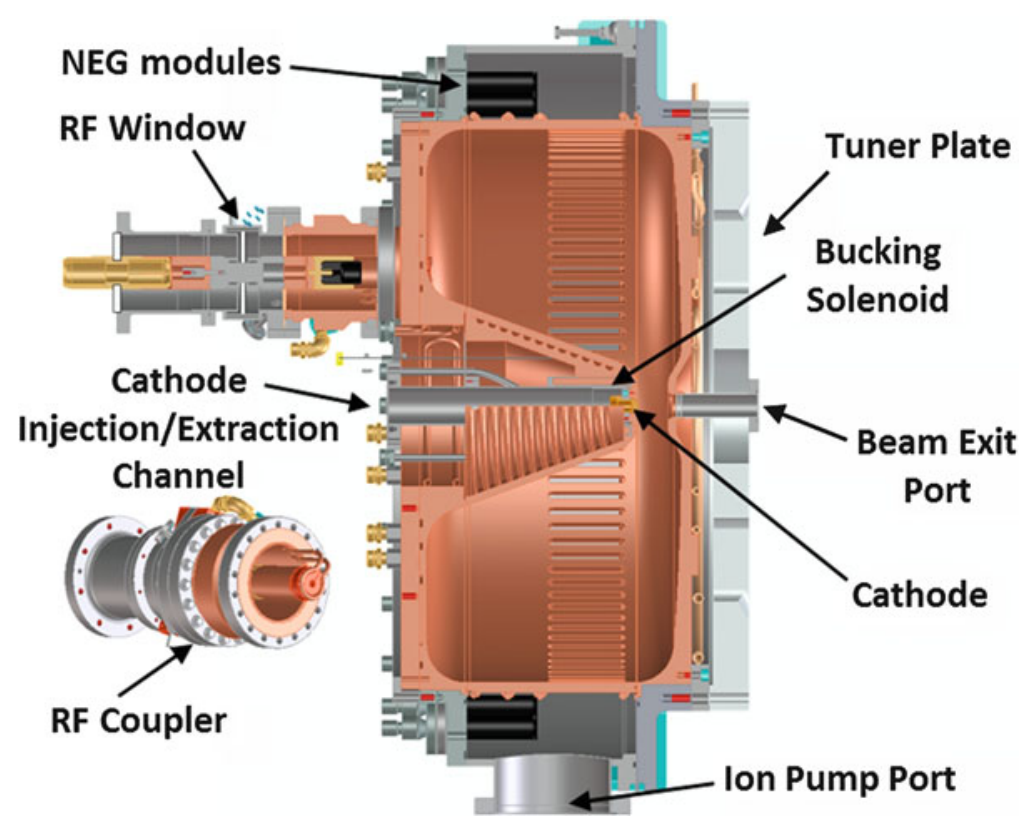

Fig. 13 APEX VHF gun cross section with main components (Figure provided by Sannibale, private communication)

normal conducting RF guns on high duty cycle (see section "NC RF Gun Results from Boeing") and beam quality (see section "Experimental Results") even further to a cavity capable of CW operation caused the choice of a low frequency, $186 \mathrm{MHz}$, NC RF gun; see Fig. 13. This low RF frequency and the corresponding large geometry of the cavity dramatically reduces the power density on the surface of the RF structure and allows CW operation with conventional water cooling techniques. The low frequency also means a lower accelerating gradient with respect to L- or S-band guns but a larger accelerating voltage as compared to DC guns. The long RF period allows the usage of photocathode laser pulses of tens of picoseconds length which reduces the space charge density. The large cavity volume ensures a good vacuum conductivity, and the long RF wavelength allows opening slots in the cavity walls, resulting in negligible field distortions to connect vacuum pumps. Low vacuum pressure during operation enables the usage of high quantum efficiency semiconductor photocathodes which are sensitive to ion back bombardment and contamination like, e.g., $\mathrm{Cs}_{2} \mathrm{Te}$ or $\mathrm{CsK}_{2} \mathrm{Sb}$ at acceptable lifetimes. This is, for example, important to reduce the average power requirements for the photocathode laser system when running in $\mathrm{CW}$ operation. To easily try out different kinds of photocathodes and to minimize the time for cathode exchange, the APEX gun design also has a load-lock system to exchange the photocathode without breaking the vacuum of the gun.

The APEX gun has been successfully commissioned (Sannibale et al. 2012) and the test facility is in continuous upgrade. The status of the experimental tests in summer 2013 and the expected performance based on simulations are summarized in column (d) of Table 3 (Sannibale, private communication). The capability to operate in $\mathrm{CW}$ mode with no problems to achieve the design accelerating fields and the design beam energy has been demonstrated. Also the good vacuum performance of about $1 \cdot 10^{-9}$ mbar while operating at nominal RF power has been proven. A $\mathrm{Cs}_{2} \mathrm{Te}$ photocathode has been used successfully to generate hundreds of $\mathrm{pC}$ per bunch at $\mathrm{MHz}$ repetition rate. Further cathode types will be tested, the next one being $\mathrm{CsK}_{2} \mathrm{Sb}$. The diagnostics for characterizing the generated electron beam is continuously extended, and in the future also 
further accelerating sections are foreseen, boosting the beam energy to $30 \mathrm{MeV}$ and allowing beam brightness measurements at reduced space charge forces.

\section{Low Average Current Electron Sources}

In this section, photo injector developments providing average beam currents of less than $1 \mu \mathrm{A}$ are presented. Two examples have been selected, the measurements or design parameters of which are listed in Table 5. The projects are described briefly in the following subsections.

Table 5 Photo injector developments for a low average current below $1 \mu$ A. For details on the projects, refer to the $\underline{\text { text }}$

\begin{tabular}{|c|c|c|c|}
\hline & (a) & (b) & (c) \\
\hline Location & LCLS, USA & \multicolumn{2}{|l|}{ SPARC_LAB, Italy } \\
\hline Gun type & NC RF gun & \multicolumn{2}{|l|}{1.6 cell NC RF Gun } \\
\hline $\begin{array}{l}\text { Experimental results or } \\
\text { design goals/simulation }\end{array}$ & Exp. results & \multicolumn{2}{|l|}{ Exp. results } \\
\hline Operation mode & & Gaussian & COMB \\
\hline Pulsed/CW & Pulsed & \multicolumn{2}{|l|}{ Pulsed } \\
\hline Cathode type & Copper & \multicolumn{2}{|l|}{ Copper } \\
\hline Single bunch charge & $20-250 \mathrm{pC}$ & Up to $1 \mathrm{nC}$ & Up to $\sim 200 \mathrm{pC}$ \\
\hline Single bunch rep rate & $120 \mathrm{~Hz}$ & $10 \mathrm{~Hz}$ & $\sim 1 \mathrm{THz}$ \\
\hline Length of bunch train & N/A & N/A & Currently $\leq 4$ pulses \\
\hline Bunch train rep rate & N/A & N/A & $10 \mathrm{~Hz}$ \\
\hline $\begin{array}{l}\text { Total beam charge gener- } \\
\text { ated per second }\end{array}$ & $2.4-30 \mathrm{nC} / \mathrm{s}$ & Up to $10 \mathrm{nC} / \mathrm{s}$ & Up to $4 \mathrm{nC} / \mathrm{s}$ \\
\hline DC voltage/gap & N/A & N/A & N/A \\
\hline Cathode peak field & $\begin{array}{l}115 \mathrm{MV} / \mathrm{m}, \\
50 \% \text { at emission }\end{array}$ & $\begin{array}{l}105 \mathrm{MV} / \mathrm{m}, \\
50 \% \text { at emission }\end{array}$ & $\begin{array}{l}100 \mathrm{MV} / \mathrm{m}, \\
50 \% \text { at emission }\end{array}$ \\
\hline Beam energy at gun exit & $6 \mathrm{MeV}$ & $\sim 5 \mathrm{MeV}$ & $4.5 \mathrm{MeV}$ \\
\hline $\begin{array}{l}\text { Norm. transv. emittance } \\
\text { (RMS) in }[\mathrm{mm} \mathrm{mrad}]\end{array}$ & $\begin{array}{lll}0.3-0.4 & \text { for } & 150 \mathrm{pC} \\
\text { at } 135 \mathrm{MeV} & & \\
\end{array}$ & $\begin{array}{l}\sim 1 \quad \text { for } \quad 280 \mathrm{pC} \\
\text { at } 147.5 \mathrm{MeV}\end{array}$ & $\begin{array}{l}0.54 \text { for } 2 \times 90 \mathrm{pC} \\
\text { at } \sim 100 \mathrm{MeV}\end{array}$ \\
\hline $\begin{array}{l}\text { Norm. transv. slice emit- } \\
\text { tance (RMS) in [mm mrad] }\end{array}$ & $\begin{array}{l}0.3-0.4 \quad \text { for } \quad 150 \mathrm{pC} \\
\text { at } 135 \mathrm{MeV} \text { (central slices) }\end{array}$ & $\begin{array}{ll}0.5-1 \quad \text { for } & 280 \mathrm{pC} \\
\text { at } 147.5 \mathrm{MeV} & \end{array}$ & N/A \\
\hline Charge fraction analyzed & $95 \%$ & $90 \%$ & $90 \%$ \\
\hline RF frequency & $2,856 \mathrm{MHz}$ & \multicolumn{2}{|l|}{$2,856 \mathrm{MHz}$} \\
\hline \multicolumn{4}{|l|}{ Photocathode laser: } \\
\hline Laser medium & Ti:Sapphire & \multicolumn{2}{|l|}{ Ti:Sapphire } \\
\hline Wavelength & $253 \mathrm{~nm}$ & \multicolumn{2}{|l|}{$266 \mathrm{~nm}$} \\
\hline Temporal pulse shape & Gaussian, 2-3 ps FWHM & $\begin{array}{l}\text { Gaussian, } \\
7.3 \text { ps FWHM }\end{array}$ & $\begin{array}{l}\mathrm{Up} \text { to } 4 \text { Gaussians } \\
(0.15 \mathrm{ps} \text { RMS }) \text { within } \\
\sim 4.3 \mathrm{ps} \\
\end{array}$ \\
\hline Transverse pulse shape & $\begin{array}{l}\text { Truncated } \text { Gaussian, } \\
\text { edge-edge } 1 \mathrm{~mm} \text { for } 150 \mathrm{pC}\end{array}$ & $\begin{array}{l}\text { Gaussian, } \\
\sigma_{x, y} \approx 0.35 \mathrm{~mm}\end{array}$ & $\begin{array}{l}\text { Gaussian, } \\
\sigma_{x, y} \approx 0.35 \mathrm{~mm}\end{array}$ \\
\hline
\end{tabular}




\section{NC RF Gun Developments for LCLS}

The BNL/SLAC/UCLA 1.6 cell S-band cavity is the most copied and operated RF gun type around the world. The LCLS project at SLAC is also based on this kind of cavity. To further improve the performance of the 1.6 cell S-band gun, several modifications to the cavity have been suggested for the LCLS project (Limborg et al. 2005). These include, for example, a larger separation between the 0 and the $\pi$ mode of the cavity. By increasing the mode separation from 3.5 to $15 \mathrm{MHz}$, the extracted emittance is slightly improved, and in particular a simplified tuning and a higher stability are expected. Another modification of the LCLS gun is a dual RF feed with coupling slots along the z-axis (beam direction) and increased radius on the inside surface of the coupler aperture. This ensures that the pulse heating should stay below $50^{\circ}$. The quadrupole moment introduced by the couplers is minimized by applying a racetrack cell shape (Xiao et al. 2005).

The LCLS gun was build, tested, and in March 2007 installed on the LCLS injector (Dowell et al. 2008). The commissioning of the injector was quick and very successful. The design goals with beam charges up to $1 \mathrm{nC}$ and a transverse projected emittance of below $1.2 \mathrm{~mm} \mathrm{mrad}$ have been demonstrated (Akre et al. 2008). The usual operation at LCLS is at lower bunch charges, e.g., with $250 \mathrm{pC}$ where transverse projected emittances down to $0.35 \mathrm{~mm}$ mrad have been measured (Emma et al. 2010), or for generating very short FEL pulses of less than $10 \mathrm{fs}$ length (Ding et al. 2009), electron bunches of $20 \mathrm{pC}$ with a transverse projected emittance of $0.19 \mathrm{~mm} \mathrm{mrad}$ are used (Frisch et al. 2010). More recent measurements are described in Zhou et al. (2012a,b, 2015). In Table 5 column (a), the typically measured injector performance at LCLS is summarized (Zhou, private communication). Please note that all the mentioned emittance data from LCLS are representative for $95 \%$ of the total bunch charge since during the analysis $2.5 \%$ of the charge in the tails are cut on each side of the projected measured beam distribution while performing the usual quad scan. The operation experience at LCLS shows that stable photocathode laser properties are of high importance for stable user operation which motivated to simplify the photocathode laser system (Zhou, private communication).

\section{NC RF Photo Injector at SPARC_LAB}

An example for the operation of a copy of the BNL/SLAC/UCLA gun in Europe is the SPARC_LAB at the INFN National Lab of Frascati (INFN-LNF) (Ferrario et al. 2013). Based on the SPARC (Alesini et al. 2003) and PLASMONX (Alesini et al. 2005) activities, the facility now called SPARC_LAB is doing multipurpose R\&D with a high brightness photo injector. On the one hand, FEL operation in SASE, seeded, and HHG modes have been performed for wavelengths from $500 \mathrm{~nm}$ down to $40 \mathrm{~nm}$. On the other hand, laser-matter interactions and especially laser-plasma acceleration experiments, e.g., for the self-injection and external injection of electrons in a plasma, are possible due to the FLAME laser system capable of generating laser pulses of up to $220 \mathrm{TW}$ and $25 \mathrm{fs}$ length. This laser system will also be used for a Thomson backscattering experiment to generate quasi coherent, monochromatic x-ray radiation. Particle-driven plasma acceleration experiments are possible using short, comb-like photocathode laser pulse trains. Column (b) of Table 5 lists some measured parameters of the electron beam for photocathode laser pulses with Gaussian temporal shape for an uncompressed electron beam (Filippetto et al. 2011; Ferrario, private communication). Column (c) lists some experimental results with a temporal comb-like photocathode laser distribution which generates short bunch trains with a micro pulse repetition rate of $\mathrm{THz}$ within the same RF pulse (Ferrario, private communication; Mostacci et al. 2011). 
Please notice that the projected emittance number quoted in column (c) is from the combined phase space of the two sub-bunches which both experience somewhat different beam dynamics.

One particularity of the photo injector setup at SPARC_LAB is that in addition to the solenoid close to the gun, the first part of the NC booster linac is embedded in an array of solenoids. Besides an extended emittance compensation and conservation, the solenoid system allows to study velocity bunching processes in order to reduce coherent synchrotron radiation (CSR) effects in downstream magnetic bunch compressors which can result in a deterioration of the beam emittance.

\section{Comparing Photo Injector Performances and Future Trends}

\section{Comparing the Experimental Emittance Results}

A comparison of different facilities can be done by plotting the single bunch emittance obtained experimentally versus the bunch charge for the different facilities (Fig. 14). To cope with the different charge fractions that have been analyzed by the different facilities, the PITZ experimental data are presented for several charge fractions (charge cuts); see section "Experimental Results." It should be noticed that the emittance values shown in this plot were obtained at different beam energies using different measurement methods.

\section{Comparing the Average Injector Brightness}

Another comparison of the different photo injector projects can be done by calculating the average brightness of the injector $B_{\text {injector }}$ (using Eq.4) for the different projects and comparing it as a function of the RF frequency for the experimental data obtained up to now. The results of such a

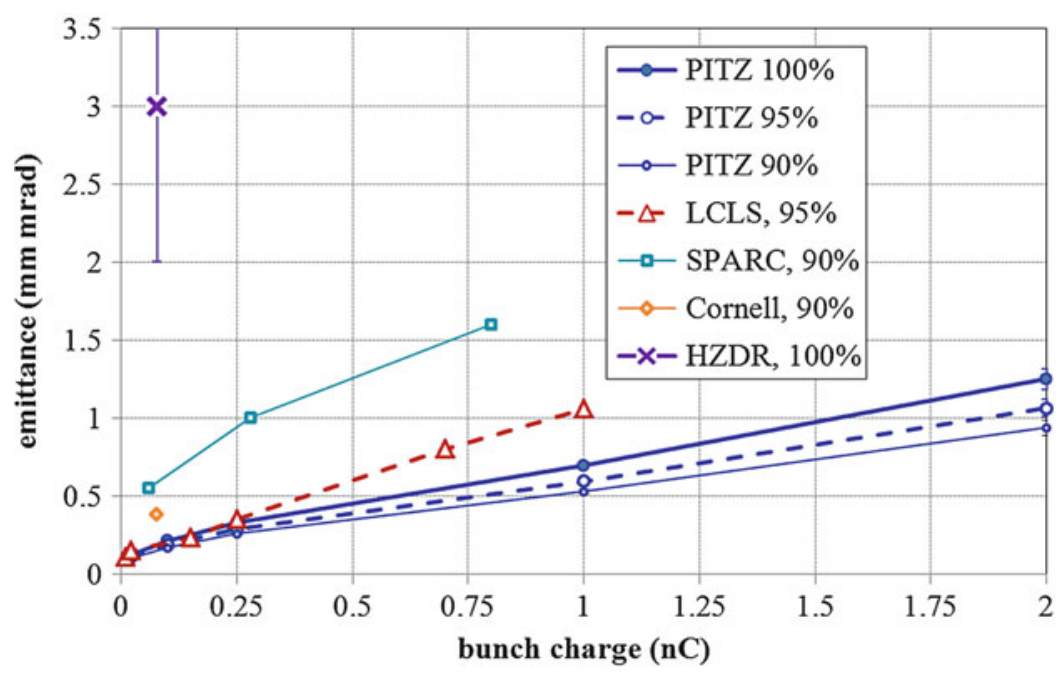

Fig. 14 Measured RMS normalized transverse emittance as a function of the bunch charge for different facilities. Presented experimental data are based on references: PITZ (Krasilnikov et al. 2012), LCLS (Akre et al. 2008; Emma et al. 2010; Frisch et al. 2010), SPARC (Filippetto et al. 2011; Cianchi et al. 2008), Cornell (Gulliford et al. 2013), and HZDR (Teichert, private communication) 


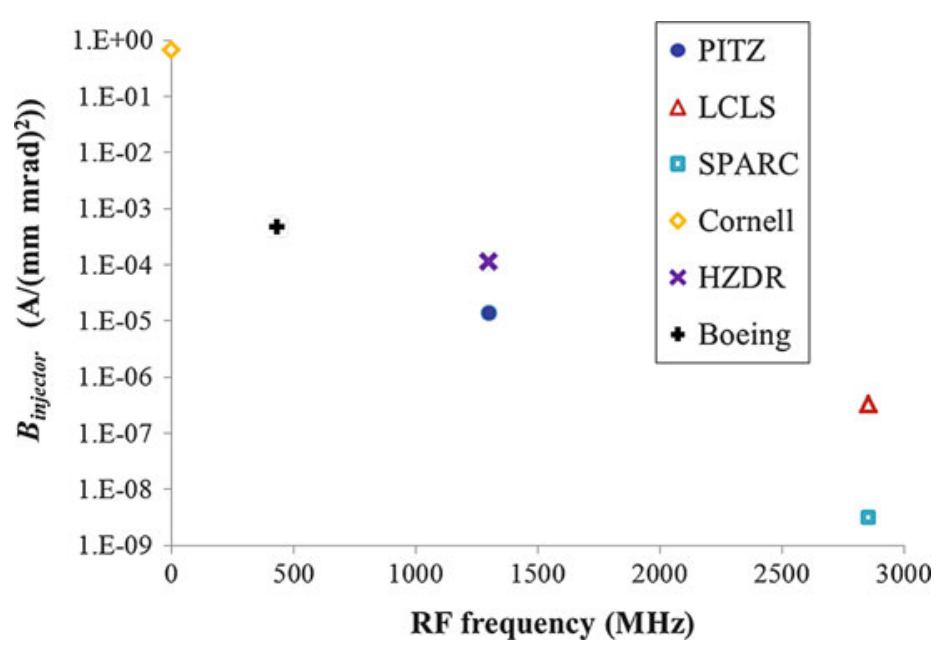

Fig. 15 Average brightness $B_{\text {injector }}$ (see Eq.4) of different photo injectors as a function of the electron source RF frequency. The values are calculated based on the best peak brightness for each facility shown in Fig. 14 in section "Comparing the Experimental Emittance Results" and applying the corresponding design average currents from the injector. Additionally the Boeing data (Dowell et al. 1993) is shown as well

comparison are shown in Fig. 15, where the design average currents from the injector $I_{\text {injector }}$ have been used for each facility. It should be noticed that this average brightness assumes the same peak (single bunch) brightness for all bunches in a train (or each bunch for CW operation). This, indeed, is not yet tested at many facilities and remains a challenge. It turns out that photo injectors with lower RF frequency tend to be able to yield higher injector brightness due to their capability to generate very high average currents.

\section{Future Trends in Photo Injector Developments}

\section{Higher Average Injector Brightness}

One future trend in photo injector developments will be the generation of higher and higher average injector brightness; see Eq. 4. This on the one hand requires higher RF duty cycles and higher bunch repetition rates, i.e., higher average beam currents, but on the other side also even better emittances in both transverse planes.

Higher Average Currents For the higher average beam currents on the one hand, developments on the photo cathode laser systems are necessary, especially when strong requirements on the shaping of the individual laser pulses have to be fulfilled in order to meet the stringent requirements on electron beam quality. For example, the development of a reliable and stable photocathode laser system generating laser bunch trains of $600 \mu \mathrm{s}$ length at $10 \mathrm{~Hz}$ repetition rate with a micro pulse repetition rate of $4.5 \mathrm{MHz}$ (i.e., 2,700 pulses per train with an UV energy of more than $3 \mu \mathrm{J}$ in each pulse) and temporal and transverse flattop shape of the individual laser pulses as required for the European XFEL is a significant development task. The development of a laser system with a micro pulse repetition rate of $3 \mathrm{MHz}$ for the FLASH FEL is described in reference (Will et al. 2011) and the temporal pulse shaping is described in Will and Klemz (2008). Significantly higher micro pulse or pulse train repetition rates will create further pulse heating problems in the laser 
system, and even more stringent requirements on the laser pulse shaping would require a different photocathode laser concept; see also the next paragraph.

On the other hand, further developments on the photocathodes (e.g., cathodes with photo emission at visible wavelengths) are needed in order to relax the requirements on the laser energy per micro pulse that has to be generated at the basic laser wavelength, i.e., the average laser power. In addition, the possibility of using, e.g., green laser light instead of the usual UV laser light also simplifies the laser system since it allows to omit the second nonlinear crystal for the UV generation which often causes significant changes of the laser pulse shape and at high laser power is also at risk of damage since high conversion efficiencies are obtained with a laser focus at the nonlinear crystal. Especially those facilities requiring high average beam currents have a strong need for new types of photocathodes, allowing for long time stable and reliable operation at visible laser wavelengths. For a discussion of cathode $R \& D$ for future light sources please see e.g. Dowell et al. 2010.

Lower Emittance The other path to obtain higher average injector brightness is to further reduce the emittance of the electron beam. One major line to do this is a further improvement of the photocathode laser pulse shaping towards the generation of 3D ellipsoidal electron bunches (Kapchinskii and Vladimirskii 1959; Serafini and Rosenzweig 1997; Limborg-Deprey 2005; Li et al. 2009). In section "Generic Photo Injector Layout and Beam Dynamics Simulations" and Table 1, it was shown that major improvements like significantly reduced slice and projected emittance and increased slice brightness can be achieved with perfect 3D ellipsoidal laser bunch shapes. In addition, further simulations have shown that the longitudinal phase space of electron beams generated with perfect 3D ellipsoidal photocathode laser distributions show an almost pure sine shape which can be corrected almost completely with a 3rd harmonic cavity so that the bunch compression can be simplified and much shorter bunch lengths can be obtained. Furthermore, the phase spaces show almost no beam halo so that the beam measurements have a better signal to noise ratio and the beam produces less radiation damage. It was also shown that the beam properties are less sensitive on the machine parameters so that a better stability is expected (LimborgDeprey 2005; Krasilnikov et al. 2013a; Khojoyan et al. 2013). Since the photo emission process and the acceleration at the cathode will modify the extracted charge distribution, a further step towards perfect electron bunch shapes can be made by pre-compensating these modifications with corresponding photocathode laser pulse shape modifications, e.g., by generating an egg-like photocathode laser pulse distribution instead of a perfect 3D ellipsoidal.

Still from the practical point of view, it is very difficult to generate such specific photocathode laser pulse shapes, especially when not only single bunches but laser bunch trains have to be generated. DESY together with the Institute of Applied Physics (IAP, Nizhny Novgorod, Russia) and the Joint Institute of Nuclear Research (JINR, Dubna, Russia) have started a development program to build a first prototype of such a photocathode laser system (Krasilnikov et al. 2013b). The laser pulse shaping is realized using a spatial light modulator technique. The pulse shaper is based on a zero dispersion optical compressor where a spectral mask can be applied to an energy-chirped laser beam after the corresponding grating. This results in a twodimensional time-space elliptically shaped laser intensity distribution. Rotating the laser beam to the perpendicular transverse plane and repeating the spectral masking yields an approximation of an 3D ellipsoidal laser pulse. Stability of the pulse shape performance as well as photon homogeneity and pulse border sharpness are the main challenges for the current developments within this project (Krasilnikov et al. 2013b; Khojoyan et al. 2013). 
It is planned that a first prototype of the 3D ellipsoidal photocathode laser system will be installed at PITZ in autumn 2014 and first beam tests should start in 2015. Since the photo injector performance is so strongly dependent on the laser shape hitting the photocathode, it is very important that not only sophisticated laser pulse shapes are generated but that such shapes can be generated with very high stability and reliability. This is especially important for user facilities like LCLS, FLASH, and European XFEL which have very stringent requirements on the beam quality and stability.

\section{A Plasma-Based Approach}

Another future trend is the worldwide interest in laser- and beam-driven plasma acceleration, and the corresponding test facilities are growing like mushrooms. While laser-plasma-generated electron beams so far typically have limited stability and produce limited beam quality (e.g., as regards energy spread and emittance) compared to the electron sources described in this chapter, there is also a proposal (Hidding et al. 2011) which tries to avoid these drawbacks by using a novel under dense plasma photocathode technique (Hidding et al. 2012). This technique uses a twocomponent gas plasma cell in which the output beam is generated by synchronized, comparably low-intensity laser pulses via tunneling ionization inside a beam-driven plasma wave. At first, a high-current electron beam (either from a state-of-the-art accelerator or a laser-plasma accelerator) is used to drive a co-moving accelerating plasma cavity by generating a plasma blowout based on the low-ionization threshold (LIT) gas component of the plasma cell like, for example, hydrogen (ionization potential $13.6 \mathrm{eV}$ ) or alkali metal vapors. This plasma cavity has a length of the order of $100 \mu \mathrm{m}$ and can produce accelerating fields of the order of tens of $\mathrm{GV} / \mathrm{m}$. The second gas component of the plasma cell (e.g., helium, ionization potential $24.6 \mathrm{eV}$ ), in contrast, remains at first in a nonionized state due to its substantially higher ionization threshold (HIT). Next, a highquality witness electron bunch will be produced by a synchronized laser pulse which is strongly focused to the ionization threshold of the HIT gas component. These HIT electrons can therefore be released directly inside the plasma wave near the plasma wake's potential minimum and are then rapidly accelerated and trapped in the accelerating phase of the plasma wave. It is expected that the beam quality of the HIT electrons inside the plasma wave is very good: When estimating their phase-space volume by the "photo cathode" laser pulse spot size and intensity, the laser contribution to the normalized emittance can be roughly approximated as $\epsilon_{n} \approx w_{0} a_{0} / 2^{1.5}$, where $w_{0}$ is the spot size and $a_{0}$ is the laser potential, which for a bunch charge of $2 \mathrm{pC}$, for example, results in $\epsilon_{n} \approx 0.03 \mathrm{~mm}$ mrad and a peak current of about $300 \mathrm{~A}$ (Hidding et al. 2012). It has to be noted, however, that the preservation of the beam quality during the extraction out of the plasma and further beam transport is challenging, as is the diagnostics of such low emittance beams. Also, the energy spread of such bunches, although much more stable and smaller than with conventional laser-plasma accelerators, is a fundamental issue especially for free-electron laser concepts. This energy spread results from the very high gradient of accelerating voltages over the plasma wave and therefore is highly correlated. Further new ideas are on the way which may reduce the energy spread towards FEL-acceptable levels in the future (Hidding, private communication).

Experimental campaigns to test this interesting idea are under preparation since 2013 and are expected to be conducted at the FACET facility at SLAC in the framework of the E-210 Trojan Horse PWFA experiment. 


\section{Summary}

There is a large variety of existing and future planned light sources, and each of them sets specific demands on the performance of their corresponding electron source. The key injector parameters characterizing the required beam properties mostly depend on user requirements at the final application and the technology choice for building the accelerator system. Therefore, there is no universal solution to build an electron source for high brightness beams applications. On the contrary, the specific requirements in many cases require individual solutions for the technical realization of the electron source.

In the introduction of this chapter, several basic parameters were introduced, and a generic photo injector layout with the corresponding physics was discussed. The European XFEL injector was used as a practical example, and the influence of the photocathode laser pulse shaping was demonstrated. Then a basic description of DC photo guns, normal conducting and superconducting RF guns followed. In the subsequent sections, several different photo injector projects with average beam current between $2 \mathrm{nA}$ and $100 \mathrm{~mA}$ were reviewed. These examples demonstrate that a large parameter space of average beam current, time structure of the beam, and beam quality can be addressed by different photo injector developments. Simulations predict very good performance of all three basic photo injector types. Major experimental progress is visible on the different subsystems (guns, laser systems, and beam diagnostics) as well as on the measured beam quality. While since the end of the 1990s "the" challenge of the photo injector community was to experimentally demonstrate normalized transverse emittances of $1 \mathrm{~mm}$ mrad for $1 \mathrm{nC}$ bunch charges, this and even better emittances have meanwhile been shown by several projects. As an example to explain the experimental emittance optimization, the photo injector test facility at DESY in Zeuthen, PITZ, was described in more detail, as it up to now has demonstrated the lowest projected emittance numbers in the bunch charge range from 0.02 to $2 \mathrm{nC}$. Since the experimental emittance results get better and better, more and more details in the technical realization, the measurement, and analysis procedure as well as other influences on the electron beam like distortions from inhomogeneous magnetic fields or wakefields have to be considered. Also research on the emission process from the cathode forming the electron beam and defining the thermal emittance as a lower limit of the transverse emittance is becoming increasingly important.

For future photo injector developments, the trend goes to even higher average beam currents from the photo injectors and ultimately low beam emittances. Some development paths that will be followed in the future have been discussed shortly.

Acknowledgments The authors would like to thank D. Dowell, B. Dunham, M. Ferrario, B. Hidding, F. Sannibale, J. Teichert, F. Zhou, and the members of the PITZ collaboration for providing material and many useful discussions. Many thanks also to K. Flöttmann and M. Gross for reading and commenting on the manuscript.

\section{Cross-References}

- Superconducting RF: Enabling Technology for Modern Light Sources 


\section{References}

R. Akre et al., Commissioning the linac coherent light source injector. Phys. Rev. ST Accel. Beams 11, 030703 (2008)

D. Alesini et al., The SPARC project: a high-brightness electron beam source at LNF to drive a SASA-FEL experiment. Nucl. Instrum. Methods A 507, 345-349 (2003)

D. Alesini et al., The project PLASMONX for plasma acceleration experiments and a Thomson X-Ray source at SPARC, in Proceedings of the PAC2005, Knoxville, 2005, pp. 820-822

A. Arnold et al., Development of a superconducting radio frequency photoelectron injector. Nucl. Instrum. Methods A 577, 440-454 (2007)

G. Asova, Tomography of the electron beam transverse phase space at PITZ, PhD thesis, Bulgarian Academy of Sciences, Sofia (2012), http://pitz.desy.de/sites/site_pitz/content/e123/e129016/ e129324/infoboxContent171923/Thesis2012Asova_eng.pdf

ASTRA Manual, update April 2014, http://www.desy.de/ mpyflo/Astra_documentation/AstraManual_V3.1.pdf

K. Baptiste et al., A CW normal-conductive RF gun for free electron laser and energy recovery linac applications. Nucl. Instrum. Methods A 599(1), 9-14 (2009). LBNL-1708E

I.V. Bazarov, C.K. Sinclair, High brightness, high current injector design for the cornell ERL prototype, in Proceedings of the PAC2003, Portland, 2003, pp. 2062-2064

I.V. Bazarov, C.K. Sinclair, Multivariate optimization of a high brightness dc gun photoinjector. Phys. Rev. ST Accel. Beams 8, 034202 (2005)

B.E. Carlsten, New photoelectric injector design for the Los Alamos National Laboratory XUV FEL accelerator. Nucl. Instrum. Methods A 285, 313-319 (1989)

B.E. Carlsten, Space charge induced emittance compensation in high brightness photoinjectors. Part. Accel. 49, 27-65 (1995)

A. Cianchi et al., High brightness electron beam emittance evolution measurements in an $\mathrm{rf}$ photoinjector. Phys. Rev. ST Accel. Beams 11, 032801 (2008)

W. Decking, T. Limberg, European XFEL Post-TDR description, XFEL.EU TN-2013-004-01, https://docs.xfel.eu/alfresco/d/a/workspace/SpacesStore/7f5974d5-23b7-48e5-a54c-a752b8376 1bc/TN-2013-004-01_TDR_Design_Changes.pdf

Y. Ding et al., Measurements and simulations of ultralow emittance and ultrashort electron beams in the linac coherent light source. Phys. Rev. Lett. 102, 254801 (2009)

D. Dowell, J. Smerge, Quantum efficiency and thermal emittance of metal photocathodes. Phys. Rev. ST Accel. Beams 12, 074201 (2009)

D.H. Dowell et al., First operation of a photocathode radio frequency gun injector at high duty factor. Appl. Phys. Lett. 63(15), 2035-2037 (1993)

D.H. Dowell, S.Z. Bethel, K.D. Friddell, Results from the average power laser experiment photocathode injector test. Nucl. Instrum. Methods A 356, 167-176 (1995)

D. Dowell et al., The development of the linac coherent light source RF Gun. ICFA Beam Dyn. Newsl. 46, 162-192 (2008)

D.H. Dowell et al., Cathode R\&D for future light sources. Nucl. Instrum. Methods A 622, 685-697 (2010)

B.M. Dunham, L.S. Cardman, C.K. Sinclair, Emmitance measurements for the illinoise/CEBAF polarized electron source, in Proceedings of the PAC1995, Dallas, 1995, pp. 1030-1032

B. Dunham et al., Record high-average current from a high-brightness photoinjector. Appl. Phys. Lett. 102, 034105 (2013) 
B. Dwersteg, K. Floettmann, J. Sekutowicz, Ch. Stolzenburg, RF gun design for the TESLA VUV Free Electron Laser. Nucl. Instrum. Methods A 393, 93-95 (1997)

P. Emma, A. Brachmann, D. Dowell et al., Beam brightness measurements in the LCLS injector, in Mini-WS on Compact X-Ray FELs Using HBB, LBNL, Berkeley, 2010

M. Ferrario, J.E. Clendenin, D.T. Palmer, J.B. Rosenzweig, L. Serafini, HOMDYN study for the LCLS RF photo-injector, in Proceedings of the 2nd ICFA Advanced Accelerator Workshop on the Physics of High Brightness Beams, UCLA, Los Angeles, 1999, pp. 534-563. SLAC-PUB-8400, 2000

M. Ferrario, K. Flöttmann, B. Grigorian, T. Limberg, Ph. Piot, Conceptual design of the XFEL photoinjector, TESLA FEL report 2001-03

M. Ferrario et al., SPARC_LAB present and future. Nucl. Instrum. Methods B 309, 183 (2013)

D. Filippetto et al., Phase space analysis of velocity bunched beams. Phys. Rev. ST Accel. Beams 14, 092804 (2011)

K. Floettmann, Note on the thermal emittance of electrons emitted by Cesium Telluride photo cathodes, TESLA-FEL report 1997-01, DESY, 1997

K. Floettmann, T. Limberg, P. Piot, Generation of ultrashot electron bunches by cancellation of nonlinear distortions in the longitudinal phase space, TESLA FEL report 2001-06

K. Flöttmann, D. Janssen, V. Volkov, Emittance compensation in a superconducting rf gun with a magnetic mode. Phys. Rev. ST Accel. Beams 7, 090702 (2004)

J. Frisch et al., Operation and upgrades of the LCLS, in Proceedings of the LINAC2010, Tsukuba, 2010, pp. 694-697

B.v.d. Geer, Conceptual Design of a 1kA, 100fs, 1 micron split RF-photoinjector, in ICFA Workshop on the Physics and Applications of High Brightness Electron Beams, Erice, 2005

C. Gulliford et al., Demonstration of low emittance in the Cornell energy recovery linac injector prototype. Phys. Rev. ST Accel. Beams 16, 073401 (2013)

B. Hidding et al., Method for generating high-energy electron beams with ultra short pulse length, width, divergence and emittance in a hybrid laser-plasma accelerator, filed as German Patent June 2011, AZ 102011104 858.1. Filed as PCT/US patent via UCLA under the title Method for Generating Electron Beams in a Hybrid Laser-Plasma-Accelerator on 18 June 2012, PCT/US Ser. No. PCT/US12/043002

B. Hidding, G. Pretzler, J.B. Rosenzweig, T. Königstein, D. Schiller, D.L. Bruhwiler, Ultracold electron bunch generation via plasma photocathode emission and acceleration in a beam-driven plasma blowout, aka Trojan horse laser electron injection and acceleration in a beam-driven plasma blowout. Phys. Rev. Lett. 108, 035001 (2012)

Z. Huang et al., Suppression of microbunching instability in the linac coherent light source. Phys. Rev. ST Accel. Beams 7, 074401 (2004)

Z. Huang et al., Measurements of the LCLS laser heater and its impact on the x-ray FEL performance, SLAC-PUB-13854, 2009

I. Isaev et al., Conditioning Status of the first XFEL gun at PITZ, in Proceedings of the FEL2013, New York, 2013, pp. 282-286

D. Janssen, V. Volkov, RF focussing - an instrument for beam quality improvement in superconducting RF guns. Nucl. Instrum. Methods A 452, 34-43 (2000)

D. Janssen, V. Volkov, Emittance compensation in a superconducting RF photoelectron gun by a magnetic RF field, in Proceedings of the EPAC2004, Lucern, 2004, pp. 330-332

D. Janssen et al., First operation of a superconducting RF-gun. Nucl. Instrum. Methods A 507, 314-317 (2003) 
D. Janssen et al., Superconducting RF guns for FELs. Nucl. Instrum. Methods A 528, 305-311 (2004)

I.M. Kapchinskii, V.V. Vladimirskii, in Proceedings of the International Conference on High Energy Access, CERN, Geneva, 1959, p. 274

M. Khojoyan et al., Beam dynamics optimization for the high brightness PITZ photo injector using 3D ellipsoidal cathode laser pulses, in Proceedings of the 35th FEL Conference, New York, 2013, p. 298

K.-J. Kim, Rf and space-charge effects in laser-driven RF electron guns. Nucl. Instrum. Methods A 275, 201 (1989)

M. Krasilnikov, Impact of the cathode roughness on the emittance of an electron beam, in Proceedings of FEL 2006 Conference, Berlin, 2006, pp. 583-586

M. Krasilnikov et al., Beam-based procedures for the RF guns, in Proceedings of the PAC2005, Knoxville, 2005, pp. 967-969

M. Krasilnikov, F. Stephan et al., Experimentally minimized beam emittance from an L-band photoinjector. Phys. Rev. ST Accel. Beams 15, 1000701 (2012)

M. Krasilnikov, F. Stephan et al., PITZ experience on the experimental optimization of the RF photo injector for the European XFEL, in Proceedings of the FEL2013, New York, 2013a, p. 160

M. Krasilnikov et al., Development of a photo cathode laser system for quasi ellipsoidal bunches at PITZ, in Proceedings of the 35th FEL Conference, New York, 2013b, p. 303

L.V. Kravchuk et al., Layout of the PITZ transverse deflecting system for longitudinal phase space and slice emittance measurements, in Proceedings of the 25th Linear Accelerator Conference, Tsukuba, 2010, p. 416

Y. Li et al., Laser pulse shaping for generating uniform three-dimensional ellipsoidal electron beams. Phys. Rev. ST Accel. Beams 12, 020702 (2009)

C. Limborg, Z. Li, L. Xiao, J.F. Schmerge, D. Dowell, S. Gierman, E. Bong, S. Gilevich, RF Design of the LCLS Gun, LCLS-TN-05-3, 2005

C. Limborg-Deprey, Maximizing brightness in photoinjectors, in Proceedings of the FEL2005, Stanford, 2005, pp. 418-421

O. J. Luiten, S.B. van der Geer, M.J. de Loos, F.B. Kiewiet, M.J. van der Wiel, How to realize uniform three-dimensional ellipsoidal electron bunches. Phys. Rev. Lett. 93, 094802-1-0948024 (2004)

D. Malyutin et al., Commissioning of new diagnostic devices at PITZ, in Proceedings of the RuPAC2012, St. Petersburg, 2012, pp. 674-676

A. Mostacci et al., Advanced beam manipulation techniques at SPARC, in Proceedings of the IPAC 2011, San Sebastian, 2011, pp. 2877-2881

D.A. Orlov, U. Weigel, D. Schwalm, A.S. Terekhov, A. Wolf, Ultra-cold electron source with a GaAs-photocathode. Nucl. Instrum. Methods A 532, 418-421 (2004)

V.V. Paramonov et al., The PITZ CDS booster cavity RF tuning and start of conditioning, in Proceedings of the 25th Linear Accelerator Conference, Tsukuba, 2010, p. 241

P. Piot, Photoinjectors R\&D for future light sources \& linear colliders, in Proceedings of the LINAC2006, Knoxville, pp. 549-553 (2006)

S. Rimjaem et al., Optimizations of transverse projected emittance at the photo-injector test facility at DESY, location Zeuthen. Nucl. Instrum. Methods A 671, 62-75 (2012)

R.A. Rimmer, A high-gradient CW RF photo-cathode electron gun for high current injectors, in Proceedings of the PAC2005, Knoxville, 2005, pp. 3049-3051 
J. Rönsch, Investigations on the electron bunch distribution in the longitudinal phase space at a laser driven RF electron source for the European X-FEL, PhD thesis, Hamburg University, 2009 and DESY-THESIS-2010-001, Jan 2010

E.L. Saldin, E.A. Schneidmiller, M.V. Yurkov, An analytical description of longitudinal phase space distortions in magnetic bunch compressors. Nucl. Instrum. Methods A 483, 516-520 (2002)

F. Sannibale et al., Advanced photoinjector experiment photogun commissioning results. Phys. Rev. ST Accel. Beams, 15, 103501 (2012)

P. Schmüser, M. Dohlus, J. Rossbach, Ultraviolet and Soft X-Ray Free-Electron Lasers (Springer, Berlin/Heidelberg, 2008), pp. 112-117

L. Serafini, M. Ferrario, Velocity bunching in photo-injectors. AIP Conf. Proc. 581, 87-106 (2001)

L. Serafini, J.B. Rosenzweig, Envelope analysis of intense relativistic quasilaminar beams in rf photoinjectors: a theory of emittance compensation. Phys. Rev. E 55, 7565-7590 (1997)

J. Staples, F. Sannibale, S. Virostek, VHF-band Photoinjector, CBP Tech Note 366, Berkeley, 26 Oct 2006

L. Staykov, Characterization of the transverse phase space at the photo-injector test facility in DESY, Zeuthen site, PhD thesis, Hamburg University, 2009, DESY-THESIS-2012-041

F. Stephan et al., New experimental results from PITZ, in Proceedings of the LINAC2008, Victoria, 2008, pp. 474-476 and references therein

F. Stephan, C.H. Boulware, M. Krasilnikov, J. Bähr et al., Detailed characterization of electron sources yielding first demonstration of European X-ray Free-Electron Laser beam quality. Phys. Rev. ST Accel. Beams 13, 020704 (2010)

J. Teichert et al., Free-electron laser operation with a superconducting radio-frequency photoinjector at ELBE. Nucl. Instrum. Methods A 743, 114-120 (2013)

The European X-Ray Free-Electron Laser, Technical design report, DESY 2006-097, July 2007

G. Vashchenko, Transverse phase space studies with the new CDS booster cavity at PITZ, PhD thesis, Hamburg University, 2013, DESY-THESIS-2013-043

V. Volkov, D. Janssen, Application of cavity transverse modes in accelerators. Phys. Rev. ST Accel. Beams, 11, 061302 (2008)

I. Will, G. Klemz, Generation of flat-top picosecond pulses by coherent pulse stacking in a multicrystal birefringent filter. Opt. Exp. 16, 14922 (2008)

I. Will, G. Koss, I. Templin, The upgraded photocathode laser of the TESLA Test Facility. Nucl. Instrum. Methods Phys. Res. A 541, 467 (2005)

I. Will, H.I. Templin, S. Schreiber, W. Sandner, Photoinjector drive laser of the FLASH FEL. Opt. Exp. 19, 23770 (2011)

D. Xiang et al., First principle measurements of thermal emittance for copper and magnesium, in Proceedings of PAC07 Conference, Albuquerque, 2007, pp. 1049-1051

L. Xiao, R.F. Boyce, D.H. Dowell, Z. Li, C. Limborg-Deprey, J.F. Schmerge, Dual feed RF gun design for the LCLS, in Proceedings of the PAC2005, Knoxville, 2005, pp. 3432-3434

F. Zhou et al., Impact of the spatial laser distribution on photocathode gun operation. Phys. Rev. ST Accel. Beams 15, 090701 (2012a)

F. Zhou et al., High-brightness electron beam evolution following laser-based cleaning of a photocathode. Phys. Rev. ST Accel. Beams 15, 090703 (2012b)

F. Zhou et al., Measurements and analyses of a collimated beam in a magnetic bunch compressor. Phys. Rev. ST Accel. Beams (2015, submitted) 